TRANSACTIONS OF THE

AMERICAN MATHEMATICAL SOCIETY

Volume 359, Number 9, September 2007, Pages 4297-4324

S 0002-9947(07)04133-5

Article electronically published on April 16, 2007

\title{
THE EVEN ISOMORPHISM THEOREM FOR COXETER GROUPS
}

\author{
M. MIHALIK
}

\begin{abstract}
Coxeter groups have presentations $\left\langle S:(s t)^{m_{s t}} \forall s, t \in S\right\rangle$ where for all $s, t \in S, m_{s t} \in\{1,2, \ldots, \infty\}, m_{s t}=m_{t s}$ and $m_{s t}=1$ if and only if $s=t$. A fundamental question in the theory of Coxeter groups is: Given two such "Coxeter" presentations, do they present the same group? There are two known ways to change a Coxeter presentation, generally referred to as twisting and simplex exchange. We solve the isomorphism question for Coxeter groups with an even Coxeter presentation (one in which $m_{s t}$ is even or $\infty$ when $s \neq t$ ). More specifically, we give an algorithm that describes a sequence of twists and triangle-edge exchanges that either converts an arbitrary finitely generated Coxeter presentation into a unique even presentation or identifies the group as a non-even Coxeter group. Our technique can be used to produce all Coxeter presentations for a given even Coxeter group.
\end{abstract}

\section{INTRODUCTION}

A Coxeter system is a pair $(W, S)$ such that $W$ is a group with Coxeter presentation $\left\langle S:(s t)^{m_{s t}} \forall s, t \in S\right\rangle$ where for all $s, t \in S, m_{s t} \in\{1,2, \ldots, \infty\}, m_{s t}=m_{t s}$ and $m_{s t}=1$ if and only if $s=t$. (The relation $(s t)^{\infty}$ means that $s t$ has infinite order in $W$.) Note that all $s \in S$ are order 2 and that if $m_{s t}=2$, then $s$ and $t$ commute.

There are two diagrams associated to a Coxeter group that appear regularly in the literature. The diagrams $V_{D}(W, S)$ and $V_{F}(W, S)$ for the Coxeter system $(W, S)$ are labeled graphs with vertex set $S$. In $V_{F}$ there is an edge labeled $m_{s t}$ between distinct vertices $s$ and $t$ if and only if $m_{s t} \neq \infty$. In $V_{D}$ there is a edge labeled $m_{s t}$ between distinct vertices $s$ and $t$ if and only if $m_{s t} \neq 2$. The vertices of components of $V_{D}$ generate factors of a direct product decomposition of $W$, and the vertices of components of $V_{F}$ generate factors of a free product decomposition of $W$. While it is traditional to call $V_{D}$ the Coxeter graph or Coxeter diagram for $(W, S)$, in this paper we only consider $V_{F}$ diagrams, and we call such diagrams Coxeter diagrams or simply diagrams.

A Coxeter presentation is even if all $m_{s t}$ for $s \neq t$ are even or $\infty$. In this case we call the corresponding Coxeter group and diagram even.

A Coxeter group is rigid if any two Coxeter presentations for this group are isomorphic presentations. In [1, Bahls shows that any Coxeter group can have

Received by the editors February 18, 2004 and, in revised form, August 6, 2005.

2000 Mathematics Subject Classification. Primary 20F55; Secondary 20 E34.

(C)2007 American Mathematical Society

Reverts to public domain 28 years from publication 
at most one even presentation. In $\S 5$, we classify the even rigid Coxeter groups. Rigidity and a variety of analogous notions are designed to give insight into a fundamental problem in the theory of Coxeter groups.

The Coxeter Isomorphism Question: Given two Coxeter presentations, do they present isomorphic groups?

The Coxeter presentations $\left\langle x, y, z: x^{2}, y^{2}, z^{2},(x y)^{3},(x z)^{2},(y z)^{2}\right\rangle$ and $\left\langle a, b: a^{2}\right.$, $\left.b^{2},(a b)^{6}\right\rangle$ present isomorphic groups, but only the latter is even. In particular, the Coxeter group presented here is not rigid.

In this paper we produce an algorithm to decide if an arbitrary Coxeter presentation presents a finitely generated even Coxeter group. Furthermore, we can decide if two (finite) Coxeter presentations present the same even Coxeter group. This solves the even Coxeter isomorphism question.

Our Theorem 7 is used by Patrick Bahl's in his thesis [1] to show that there is a unique even Coxeter presentation for a finitely generated even Coxeter group. We in turn use Bahl's result in the final stage of our algorithm to decide if two finite Coxeter presentations present the same even Coxeter group. The proof of a vital combinatorial lemma hinges on the visual decomposition theorem of [7]. A critical tool in our algorithm is that of twisting in Coxeter diagrams. This method of producing different Coxeter diagrams (and different presentations) for the same Coxeter group was introduced by N. Brady, J. McCammond, B. Mühlherr and W. Neumann in 5. At this time the only known way to produce different Coxeter diagrams for a given Coxeter group is by twisting or simplex exchange.

Our main theorem is the following:

Theorem 1. Suppose $(W, S)$ is an even Coxeter system and $V^{\prime}$ is a Coxeter diagram for $W$ with odd labeled edge $[x y]$. Then there is a diagram $V^{\prime \prime}$ for $W$ obtained from $V^{\prime}$ by first performing a twist around $[x y]$ and then replacing a triangle [xyu] by an edge with an even label.

The proof of our theorem specifically defines the set to be twisted and a vertex $u$ so that triangle $[x y u]$ may be replaced by an even edge. The resulting diagram for $W$ has (one) fewer odd labeled edges than the original. Thus we have a simple algorithm to change a non-even diagram for a finitely generated even Coxeter group $W$ into the unique even diagram for $W$.

If $(W, S)$ is an arbitrary Coxeter system with diagram $V$ containing an odd edge $[x y]$, then either the described twist and triangle replacement can be carried out or $W$ is not an even Coxeter group. Hence one can decide if a given finitely generated Coxeter group is even or not. Given two finitely generated Coxeter systems $\left(W_{1}, S_{1}\right)$ and $\left(W_{2}, S_{2}\right)$ with diagrams $V_{1}$ and $V_{2}$ respectively, one can decide if $W_{1}$ and $W_{2}$ are isomorphic even Coxeter groups. Simply apply our algorithm repeatedly to $V_{1}$ and $V_{2}$ until either an odd edge cannot be replaced by an even one using our technique (in which case one of the groups is not even), or until all odd edges are replaced in both diagrams. In the latter scenario, Bahls' even rigidity result implies that the resulting even diagrams are diagram isomorphic if and only if $W_{1}$ and $W_{2}$ are isomorphic.

It is also evident that given a Coxeter system for a finitely generated even Coxeter group, one can use the methods of this paper to produce all other Coxeter systems for that group. 


\section{Preliminaries}

In this section, we describe: twisting in Coxeter diagrams as introduced in [5], visual decompositions of Coxeter groups [7, and techniques to construct quotient maps of Coxeter groups that match quotient maps of Coxeter diagrams.

(1) Twisting. In an arbitrary Coxeter system $(W, S)$, twisting makes sense for any subset of $S$ that generates a finite subgroup of $W$. We only need twist around pairs of distinct vertices $x, y \in S$ such that $m_{x y}$ is an odd integer.

Suppose $V$ is a Coxeter diagram for the Coxeter system $(W, S)$. Given $x, y \in S$, define $l k(x)$ (the "link" of $x$ ) to be the set of all vertices of $V$ that are connected to $x$ by an edge. Define $l k_{2}(x)$ (the "2-link" of $x$ ) to be the set of all vertices of $V$ that are connected to $x$ by an edge labeled 2. Define st $(x)$ (the "star" of $x$ ) to be $l k(x) \cup\{x\}$. Define $l k_{2}(x, y)$ (the "2-link" of $x$ and $y$ ) to be $l k_{2}(x) \cap l k_{2}(y)$, i.e. the set of all vertices in $V$ that are connected to both $x$ and $y$ by an edge labeled 2. So each $s \in l k_{2}(x, y)$ commutes with both $x$ and $y$. Denote $b^{-1} a b$ by $a^{b}$. Now suppose $x$ and $y$ are distinct elements of $S$ and $m_{x y}=2 n+1$. Let $d$ be the (unique) element of length $2 n+1$ in $\langle x, y\rangle$. Note that $x^{d}=y$ and $y^{d}=x$. Suppose $U \subset S-\{x, y\}$ and for each edge $[u s]$ such that $s \in S-(U \cup\{x, y\})$ and $u \in U, s \in l k_{2}(x, y)$. Then the twisting theorem of [5] implies that $\left(W, S^{\prime}\right)$ is a Coxeter system, where $S^{\prime}=U^{d} \cup(S-U)$ and a diagram for $\left(W, S^{\prime}\right)$ is obtained from $V$ by changing each edge of $V$ that connects a vertex $u \in U$ to a vertex $v \in\{x, y\}$ to connect instead from $u$ to $v^{d}$, and leaving other edges unchanged.

(2) Visual decompositions of Coxeter groups. Suppose $V$ is the diagram for a Coxeter system $(W, S)$ and some subset $C$ of $S$ separates vertices of $V$. Then a simple examination of presentations, shows that $W$ decomposes as $\langle A\rangle *\langle C\rangle\langle B\rangle$, where $A \cup B=S, A$ is $C$ union the vertices of some set of components of $V-C$ and $B$ is $C \cup(S-A)$. This type of decomposition extends in a natural way to graphs of groups decompositions of $W$. Such decompositions are called "visual" decompositions of $W$ since they are easily seen in $V$ and the main theorem of [7] states that given any graph of groups decomposition of $W$ there is a visual decomposition that basically refines the given decomposition. More specifically, any vertex (edge) group of the visual decomposition of $W$ is a subgroup of a conjugate of a vertex (edge) group of the given decomposition. For our purposes this result is particularly useful when we have two different diagrams for $W$ so that visual decompositions with respect to the two diagrams can be played against one another.

(3) Coxeter quotients. Suppose $(W, S)$ is a Coxeter system with diagram $V$. If $T \subset W$, then let $N(T)$ be the normal closure of $T$ in $W$. If $T \subset S$, then $W / N(T)$ is a Coxeter group with diagram obtained from $V$ by removing the vertices of $T$ and all vertices that connect to a vertex of $T$ by a path with all odd labeled edges. In this paper, we often consider a diagram for an even Coxeter system $(W, S)$ and another diagram $V^{\prime}$ for the system $\left(W, S^{\prime}\right)$ where $V^{\prime}$ may have odd labeled edges. Our Theorem 7 describes a 1-1 correspondence between the set of edges with label $>2$ in $V$ and those edges with label $>2$ in $V^{\prime}$. If an edge [xy] of $V^{\prime}$ has odd label and $[x y]$ corresponds to the edge $[a b]$ of $V$, then in fact, the cyclic group $\langle x y\rangle$ is conjugate to the group $\left\langle(a b)^{2}\right\rangle$. A diagram for $W / N(x y)$ is obtained from $V^{\prime}$ by collapsing the edge $[x y]$ to a point. If $[x y u]$ is a triangle, then our Proposition 2 states that $[x u]$ and $[y u]$ are labeled 2. Hence additional "collapsing" in $V^{\prime}$ is not generated by the collapsing of $[x y]$ (see Lemma 8). A diagram for $W / N(x y)$ is obtained from $V$ by changing the label on $[a b]$ to 2 . In this way, we can be sure 
that $W / N(x y)$ is an even Coxeter group with a diagram that preserves potentially desirable aspects of $V^{\prime}$. Other quotients of diagrams for $(W, S)$ and $\left(W, S^{\prime}\right)$ are obtained when we find subsets $\sigma \subset S$ and $\sigma^{\prime} \subset S^{\prime}$ such that $\langle\sigma\rangle$ and $\left\langle\sigma^{\prime}\right\rangle$ are conjugate. If $f:\left\langle\sigma^{\prime}\right\rangle \rightarrow \mathbb{Z}_{2}(=\{-1,1\})$ is a homomorphism, and $N$ is the normal closure in $W$ of $\operatorname{ker}(f)$, then Lemma 18 describes how to obtain an even diagram for $W / N$ from $V$. Understanding how quotients of $W$ correspond to quotients of two different diagrams for $W$ is crucial to the success of our arguments in this paper.

\section{A REDUCtion AND OUtLINE}

First some terminology. An edge loop in a diagram that does not cross itself is a circuit. An edge connecting two non-consecutive vertices of a circuit is a chord.

The proof of the main theorem can be easily derived from the following three propositions.

Proposition 2. Suppose $W$ is a finitely generated even Coxeter group. If $V^{\prime}$ is a diagram for $W$ with odd labeled edge [xy], then any triangle of $V^{\prime}$ containing [xy] has two edges labeled 2.

Proposition 3. Suppose $V$ is an even diagram for the Coxeter group $W,[x y]$ is an odd edge in a diagram $V^{\prime}$ for $W$ and every edge $[x c]$ of $V^{\prime}$ for $c \neq y$ is such that $c \in l k_{2}(x, y)$. Then there exists a vertex $u \in l k_{2}(x, y)$ such that if $[u c]$ is an edge with $c \notin\{x, y\}$, then [uc] has label 2, $c \in l k_{2}(x, y)$, and any simplex $\sigma^{\prime}$ of $V^{\prime}$ that contains $u$ (respectively $x$ and $y$ ) and is such that $\left\langle\sigma^{\prime}\right\rangle$ is conjugate to $\langle\sigma\rangle$ for $\sigma$ a simplex of $V$, contains $\{x, y\}$ (respectively $u$ ).

Proposition 4. If $W$ is a finitely generated even Coxeter group and $V^{\prime}$ is a diagram for $W$, then every circuit in $V^{\prime}$ of length at least 4 and containing an odd labeled edge, has a chord.

The proofs of these propositions will be given in Section 4 (Proposition 2), Section 6 (Proposition 3), Section 7 (base case of Proposition 4) and Section 8 (inductive step of Proposition 44).

In the remainder of this section we apply these three propositions to prove:

Proposition 5. Suppose $W$ is a finitely generated even Coxeter group and $V^{\prime}$ is a diagram for $W$ with odd labeled edge $[x y]$ such that every circuit containing $[x y]$ of length $\geq 4$ has a chord, then after a twist, a triangle containing [xy] can be replaced by an edge with even label. (In particular, the main theorem is reduced to

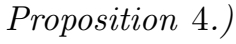

Proof. Our hypothesis and Proposition 2 imply:

Claim 5.1. Every path in $V^{\prime}$ from $x$ to $y$ either contains the edge $[x y]$ or intersects $l k_{2}(x, y)$.

Suppose $U$ is the union of all components $C$ of $V^{\prime}-\left(\{x, y\} \cup l k_{2}(x, y)\right)$ such that there is an edge from $x$ to $C$. Then by Claim 5.1, there is no edge from $y$ to $U$. If there is a vertex $t \in V^{\prime}-(\{x, y\} \cup U)$ that connects to $U$ by an edge, then $t \in l k_{2}(x, y)$ and so $U$ can be twisted around $\{x, y\}$, to form the diagram $\hat{V}^{\prime}$ for $W$. Note that after twisting, vertices of $U$ that were connected to $x$ are replaced in $\hat{V}^{\prime}$ by vertices that connect to $y$ instead. I.e. in $\hat{V}^{\prime}$, each edge (other than $[x y]$ ) containing $x$ has its other vertex in $l k_{2}(x, y)$. 
Proposition 3 implies that the triangle $[x y u]$ of $\hat{V}^{\prime}$ can be replaced by the edge $[y b]$ with label 2 times the order of $x y$, finishing Proposition 5 . More specifically, form the diagram $\tilde{V}^{\prime}$ from $\hat{V}^{\prime}$ by removing the vertices $x$ and $u$, adding a vertex $b$ and edge from $y$ to $b$ with label 2 times the order of $x y$, and for each vertex $c$ of $l k(x)-\{u, y\}=l k(u)-\{x, y\}$, add an edge labeled 2 from $c$ to $b$. So $\hat{V}^{\prime}$ is a diagram for $W$ satisfying the conclusion of Proposition 5 and $\tilde{V}^{\prime}$ has fewer odd edges than $V^{\prime}$.

\section{A matching theorem and the proof of Proposition 2}

We show that given two diagrams for an even Coxeter group, there is a bijection between the set of edges with labels greater than 2 of one diagram and that set of edges of the other diagram. The bijection is such that the commutator subgroups of the groups generated by the vertices of matching edges are conjugate. This result is then used to prove Proposition 2, Through the remainder of the paper we rely on 4 as a reference for basic facts about Coxeter groups.

If $V$ is a diagram for a Coxeter group $W$, then a simplex $\sigma$ is spherical if $\langle\sigma\rangle$ is a finite subgroup of $W$ and $\sigma$ is maximal spherical if $\sigma$ is spherical and properly contained in no other spherical simplex. Maximal spherical simplices of $V$ give (up to conjugation) the maximal finite subgroups of $W$. Hence if $V$ and $V^{\prime}$ are diagrams for $W$ and $\sigma$ is a maximal spherical simplex of $V$, then there is a maximal spherical simplex $\sigma^{\prime}$ of $V^{\prime}$ such that $\langle\sigma\rangle$ is conjugate to $\left\langle\sigma^{\prime}\right\rangle$. In [7], the groups generated by maximal simplices of $V$ are shown to be (up to conjugation) the maximal FA subgroups of $W$. Hence if $\sigma$ is a maximal simplex of $V$, then there is a maximal simplex $\sigma^{\prime}$ of $V^{\prime}$ such that $\langle\sigma\rangle$ is conjugate to $\left\langle\sigma^{\prime}\right\rangle$. In [9], Serre shows that $\langle\sigma\rangle$ is FA for any simplex $\sigma$ of $V$. In particular, for any graph of groups decomposition of $W,\langle\sigma\rangle$ is a subgroup of a conjugate of a vertex group of the decomposition.

If a triangle $[x y z]$ in a diagram $V$ for a Coxeter group has edge labels $(a, b, c)$, then $\langle x, y, z\rangle$ is finite if and only if $\frac{1}{a}+\frac{1}{b}+\frac{1}{c}>1$. A result of Tits (see [4]) implies that if $A$ is a finite subgroup of a Coxeter group $W$, and $(W, S)$ is a Coxeter system, then there is a subset $T$ of $S$ such that $\langle T\rangle$ is finite and $A$ is a subgroup of a conjugate of $\langle T\rangle$. If $(W, S)$ is even, and $T \subset S$ is such that $\langle T\rangle$ is finite, then $\langle T\rangle$ decomposes as a direct product of groups each factor which is dihedral or $\mathbb{Z}_{2}$. It is straightforward to see that none of the finite triangle groups $(2,3,3),(2,3,4)$ and $(2,3,5)$ are isomorphic to a subgroup of a direct product of dihedral groups. Tits' result then implies that these finite triangle groups are not subgroups of an even Coxeter group.

Remark 1. For any integer $n, D_{n} \equiv\left\langle u, v: u^{2}, v^{2},(u v)^{n}\right\rangle$. The commutator subgroup of $D_{n}$ is generated by $u v$ if $n$ is odd and by $(u v)^{2}$ if $n$ is even. We have $D_{2(2 k+1)} \equiv\left\langle u, v: u^{2}, v^{2},(u v)^{2(2 k+1)}\right\rangle=\langle u, v u v\rangle \times\left\langle(u v)^{2 k+1}\right\rangle \equiv D_{2 k+1} \times \mathbb{Z}_{2}$. In this case, the commutator subgroups of $\langle u, v\rangle$ and $\langle u, v u v\rangle$ are equal and generated by $(u v)^{2}$. If $n$ is not of the form $2(2 k+1)$, then $D_{n}$ is irreducible (with respect to direct product decompositions). There are other finite "basic" Coxeter groups that decompose as a direct product of $\mathbb{Z}_{2}$ and another Coxeter group, but none of these are subgroups of an even Coxeter group.

Lemma 6. Suppose the group $G$ decomposes as direct products $\prod_{i=1}^{q} A_{i}=\prod_{i=1}^{q} B_{i}$ where each $A_{i}$ and $B_{i}$ is either $\mathbb{Z}_{2}$ or $D_{k}$ for $k \neq 2(2 m+1)$ (i.e. $D_{k}$ is an irreducible 
dihedral group). If $B_{i}=\left\langle x, y: x^{2}, y^{2},(x y)^{n}\right\rangle$, then there exists a unique integer $j$ such that $A_{j}=\left\langle u, v: u^{2}, v^{2},(u v)^{n}\right\rangle$ and

(i) for odd $n$, there exists a $p$ such that $x y=(u v)^{p}$ and $\langle x y\rangle=\langle u v\rangle$,

(ii) for even $n$, there exists a $p$ and $t$ such that $x y=(u v)^{p} t$ where $t$ has order $\leq 2$ and commutes with $u$ and $v$ and $\left\langle(x y)^{2}\right\rangle=\left\langle(u v)^{2}\right\rangle$.

In particular, the commutator subgroups of $B_{i}$ and $A_{j}$ agree.

Proof. In either case, for all $t \in G, t x y t^{-1}=(x y)^{ \pm 1}$. Say $x y=a_{1} \cdots a_{q}$ where $a_{i} \in A_{i}$. Since $x y$ does not have order 2 , we may assume that $a_{1}$ has order greater than 2. Say $A_{1}=\left\langle u, v: u^{2}, v^{2},(u v)^{m}\right\rangle$. Then $a_{1}=(u v)^{p}$ and $(x y)^{ \pm 1}=u(x y) u=$ $a_{1}^{-1} a_{2} \cdots a_{q}$. As $a_{1} \neq a_{1}^{-1}$, we must have $a_{i}=a_{i}^{-1}$ for all $i \geq 2$. If $x y$ has odd order, then each $a_{i}$ is trivial or has odd order. In this case, $a_{i}=1$ for all $i \geq 2$ and $x y=a_{1}$. If $n$ is even, then $x y=a_{1} t$ where $t$ has order 2 and commutes with $u$ and $v$.

In any case, the cyclic group $\langle x y\rangle$ is normal in $G$ and the quotient of $G$ by $\langle x y\rangle$ has irreducible decomposition obtained from $\prod_{j=1}^{q} B_{j}$ by replacing $B_{i}$ by $\mathbb{Z}_{2}$. Suppose $n$ is odd, and $a_{1}=(u v)^{p}$. By the Krull-Schmidt theorem (see [10]), $\left\langle(u v)^{p}\right\rangle$ must have index 2 in $A_{1}$ and so $\left\langle(u v)^{p}\right\rangle=\langle u v\rangle$ as desired. This implies that $u v$ and $x y=(u v)^{p}$ have the same order and so $m=n$.

Now suppose $n$ is even. In this case, $(x y)^{2}=a_{1}^{2}=(u v)^{2 p}$. The quotient of $G$ by the normal subgroup $\left\langle(x y)^{2}\right\rangle$ has decomposition obtained from $\prod_{j=1}^{q} B_{j}$ by replacing $B_{i}$ by $\mathbb{Z}_{2} \times \mathbb{Z}_{2}$. Hence the quotient of $A_{1}$ by $\left\langle(u v)^{2 p}\right\rangle$ is $\mathbb{Z}_{2} \times \mathbb{Z}_{2}$. If $m$ is odd, then $D_{m}$ does not map onto $\mathbb{Z}_{2} \times \mathbb{Z}_{2}$ so $u v$ has even order. If $(u v)^{2} \notin\left\langle(u v)^{2 p}\right\rangle$, then $1, u, u v,(u v)^{2}$ and $(u v)^{3}$ would represent 5 different $(u v)^{2 p}$-cosets of $\langle u, v\rangle$ which is impossible. Hence $\left\langle(u v)^{2 p}\right\rangle=\left\langle(u v)^{2}\right\rangle$. This implies that $x y$ and $u v$ have the same order and so $m=n$.

In either case $\left\langle(x y)^{2}\right\rangle=\left\langle(u v)^{2}\right\rangle$. The uniqueness follows by construction.

Remark 2. If $V$ is a diagram for a Coxeter system $(W, S)$ and $[a b]$ is an even labeled edge of $V$, then a simple examination of presentations shows that $W / N\left(\left(a b^{2}\right)\right)$ (the quotient of $W$ by the normal closure of $\left\{(a b)^{2}\right\}$ ) is a Coxeter group with a diagram obtained from $V$ by changing the label of $[a b]$ to 2 . The subgroup $\langle S-\{t\}\rangle$ for $t \in\{a, b\}$ injects under the quotient.

Theorem 7. Suppose $W$ is a finitely generated even Coxeter group with diagrams $V$ and $V^{\prime}$ (not necessarily even). There is a unique bijection $\alpha$ between the edges [xy] of $V^{\prime}$ with label $>2$ and the edges $[a b]$ of $V$ with label $>2$ such that if $\alpha([x y])=[a b]$, then the commutator subgroup of $\langle x, y\rangle$ is conjugate to the commutator subgroup of $\langle a, b\rangle$.

Proof. Observe that the theorem can be reduced to the case when all edges of $V$ have even labels. So we make that assumption. Let $\sigma^{\prime}$ be a maximal spherical simplex of $V^{\prime}$ containing $[x y]$ and $\sigma$ the maximal spherical simplex of $V$ such that $\left\langle\sigma^{\prime}\right\rangle$ is conjugate to $\langle\sigma\rangle$. By conjugating, we assume $\left\langle\sigma^{\prime}\right\rangle=\langle\sigma\rangle$. Let $e_{1}^{\prime}, \ldots, e_{n}^{\prime}$ be the edges of $\left\langle\sigma^{\prime}\right\rangle$ not labeled by 2 . Then $\left\langle\sigma^{\prime}\right\rangle$ naturally decomposes as a direct product $A^{\prime} \cong \prod_{i=1}^{m} A_{i}^{\prime}$ and for $i \in\{1, \ldots, n\}, A_{i}^{\prime}$ is the dihedral group $D_{k_{i}}$ where $k_{i}$ is the label of $e_{i}^{\prime}$ when $e_{i}^{\prime}$ has an odd label or a label a multiple of 4 , and $k_{i}$ is half the label of $e_{i}^{\prime}$ if $e_{i}^{\prime}$ has a label two times an odd. So if $e_{i}^{\prime}=[x y]$ has a label 2 times an odd integer $q$, then $q=k_{i}$ and $D_{2 q}=\langle x, y\rangle$ decomposes as in Remark 1 as $\mathbb{Z}_{2} \times D_{q}=\left\langle(x y)^{q}\right\rangle \times\langle x, y x y\rangle$. All other $A_{j}^{\prime}$ are copies of $\mathbb{Z}_{2}$. Similarly decompose 
$\langle\sigma\rangle$ as the direct product $A \cong \prod_{i=1}^{m} A_{i}$. (Note that the number of factors in the decompositions of $\langle\sigma\rangle$ and $\left\langle\sigma^{\prime}\right\rangle$ are the same, and there is a bijection $\phi$ of the set of $A_{i}^{\prime}$ to the set of $A_{i}$ such that $\phi\left(A_{i}^{\prime}\right)$ is isomorphic to $A_{i}^{\prime}$, by Krull-Schmidt.)

Now apply Lemma 6 to get a map $\alpha$ from the edges with label $>2$ of $V^{\prime}$ to those of $V$. If $[a b]$ and $[c d]$ are distinct edges of $V$ with labels $>2$, then $\left\langle(a b)^{2}\right\rangle$ is not conjugate to $\left\langle(c d)^{2}\right\rangle$ since $\left\langle(c d)^{2}\right\rangle$ injects under the quotient of $W$ by $N\left((a b)^{2}\right)$ (see Remark 2). Hence there is exactly one choice for $\alpha$. By considering maximal spherical simplices in $V$, we see that $\alpha$ is onto.

It remains to show that $\alpha$ is injective. I.e. if $[x y]$ and $[s t]$ are edges of $V^{\prime}$, then the commutator subgroup of $\langle x, y\rangle$ and $\langle s, t\rangle$ are not conjugate. Otherwise, we see that for some $w \in W,\langle x, y\rangle \cap w\langle s, t\rangle w^{-1}$ contains the commutator subgroup of $\langle x, y\rangle$. Now, $\langle x, y\rangle \cap w\langle s, t\rangle w^{-1}=u\langle T\rangle u^{-1}$ for $T \subset\{x, y\}$ and $u \in\langle x, y\rangle$. Hence $T=\{x, y\}$. Let $S^{\prime}$ be the vertex set of $V^{\prime}$ so $V^{\prime}$ is the diagram of the Coxeter system $\left(W, S^{\prime}\right)$. It is well understood [6] when $\langle x, y\rangle$ can be conjugate to $\langle s, t\rangle$ for $\{x, y\} \neq\{s, t\}$. In particular, there must be sets $T_{i} \subset S$ for $i \in\{1, \ldots, n\}$ and $\left\{s_{i}, t_{i}, s_{i+1}, t_{i+1}\right\} \subset T_{i}$ such that $\left\langle T_{i}\right\rangle$ is finite, $\{s, t\}=\left\{s_{1}, t_{1}\right\},\left\{s_{i}, t_{i}\right\}=$ $a_{i}\left\{s_{i+1}, t_{i+1}\right\} a_{i}^{-1}$ for some $a_{i} \in\left\langle T_{i}\right\rangle$ and $\{x, y\}=\left\{s_{n+1}, t_{n+1}\right\}$. But as $\left\langle T_{1}\right\rangle$ has a direct product decomposition such that each factor is dihedral or $\mathbb{Z}_{2},\{s, t\}$ cannot be conjugated off of itself by an element of $\left\langle T_{1}\right\rangle$. Hence $\alpha$ is injective.

In the preprint [8], we generalize Theorem 7 from even Coxeter groups to general Coxeter groups and from dihedral groups to noncyclic, maximal, finite, irreducible, visual subgroups.

Proof of Proposition 2. Suppose $W$ is a finitely generated even Coxeter group, $V^{\prime}$ is a diagram for $W$ with odd labeled edge $[x y]$ and $[x y z]$ is a triangle in $V^{\prime}$. We wish to show this triangle has two edges labeled 2. Let $V$ be an even diagram for $W$. Suppose [xy] is labeled $2 k+1$ and corresponds to the edge [ab] of $V$. Also assume that $[x z]$ is labeled $m>2$ and corresponds to $[c d] \neq[a b]$. Then either $[z y]$ is labeled 2 , or $[z y]$ corresponds to $[e f] \notin\{[c d],[a b]\}$. Observe that $N(x y)=N\left((a b)^{2}\right)$. We consider the quotient map $q: W \rightarrow W / N(x y)$ and observe that $\langle c, d\rangle$ injects under $q$. (A diagram for $W / N(x y)=W / N\left(\left(a b^{2}\right)\right.$ ) is obtained from $V$ by changing the label of $[a b]$ to 2 .)

If $[z y]$ is labeled 2, then $(x z)^{2} \in \operatorname{ker}(q)$. Either $\langle x z\rangle$ is conjugate to $\left\langle(c d)^{2}\right\rangle$ and $(c d)^{2}$ has odd order, or $\left\langle(x z)^{2}\right\rangle$ is conjugate to $\left\langle(c d)^{2}\right\rangle$. In the first case, $(c d)^{4}$ is in $\operatorname{ker}(q)$ which is impossible. In the second case, $(c d)^{2}$ is in $\operatorname{ker}(q)$, which is also impossible. We conclude $[z y]$ does not have label 2 .

If $[y z]$ corresponds to $[e f]$, then again let $q: W \rightarrow W / N(x y)=W / N\left((a b)^{2}\right)$ be the quotient map. We have $q(z x)=q(z y)$. If the labels of $[x z]$ and $[z y]$ are odd, then $q\left(\left\langle(c d)^{2}\right\rangle\right)=q(\langle x z\rangle)=q(\langle y z\rangle)=q\left(\left\langle(e f)^{2}\right\rangle\right)$. But this is impossible as $q\left(\left\langle(c d)^{2}\right\rangle\right) \neq q\left(\left\langle(e f)^{2}\right\rangle\right)$ in $W / N\left((a b)^{2}\right)$. If the labels of $[x z]$ and $[z y]$ are even, then $q\left(\left\langle(c d)^{2}\right\rangle\right)=q\left(\left\langle(x z)^{2}\right\rangle\right)=q\left(\left\langle(z y)^{2}\right\rangle\right)=q\left(\left\langle(e f)^{2}\right\rangle\right)$ which is again impossible. If the label of $[x z]$ is odd and the label of $[z y]$ is even, then $q\left(\left\langle(c d)^{4}\right\rangle\right)=q\left(\left\langle(x z)^{2}\right\rangle\right)=$ $q\left(\left\langle(z y)^{2}\right\rangle\right)=q\left(\left\langle(e f)^{2}\right\rangle\right)$. Again, $q\left(\left\langle(c d)^{4}\right\rangle\right) \neq q\left(\left\langle(e f)^{2}\right\rangle\right)$ in $W / N\left((a b)^{2}\right)$. Similarly if the label of $[x z]$ is even and the label of $[z y]$ is odd.

If $[x y]$ is an edge in a general diagram of a Coxeter group $W$, the quotient group $W / N(x y)$ is a Coxeter group with a diagram obtained by first collapsing the edge $[x y]$ and then collapsing other edges and identifying other pairs of edges as a 
consequence of the collapse of $[x y]$. As a direct consequence of Proposition 2 we have the following result that will be useful throughout the remainder of the paper:

Lemma 8. Suppose $W$ is a finitely generated even Coxeter group and $V^{\prime}$ is a diagram with odd labeled edge $[x y]$. Then the diagram for $W / N(x y)$ obtained from $V^{\prime}$ by collapsing the edge $[x y]$ is such that no other edge of $V^{\prime}$ is collapsed and the only edges of $V^{\prime}$ that are identified are those in a triangle containing $[x y]$.

\section{Classifying the Rigid even Coxeter groups}

In this section we develop several propositions and lemmas, and end with a proof of Theorem 17, a classification of the rigid even Coxeter groups. Several of our arguments are based on minimal counterexample ideas. The following definition is used extensively. For a Coxeter diagram $V$, let $T(V)$ be the product of all edge labels of $V$.

The Deletion Condition for Coxeter groups implies the following:

Lemma 9. Suppose $(W, S)$ is a Coxeter system, $\Gamma$ the Cayley graph of $W$ with respect to $S$ and $T \subset S$. If $u$ and $v$ are vertices of $\Gamma$ (i.e. elements of $W$ ), then there is a unique closest vertex $w$ of the coset $v\langle T\rangle$ to $u$. Furthermore, if $\alpha$ is a geodesic from $u$ to $w$, and $\beta$ is a geodesic at $w$ in the letters of $T$, then $\alpha \beta$ is geodesic.

Proposition 10. Suppose $(W, S)$ is an even Coxeter system, $a, b \in S$ and ab has finite order $>2$. If $y \in W$ is such that $y$ conjugates $(a b)^{2}$ to $(a b)^{ \pm 2}$, then $y$ can be written geodesically as uv, where $u \in\langle a, b\rangle$ and $v \in l k_{2}(a, b)$.

Proof. We first show that $y$ conjugates $\langle a, b\rangle$ to itself. We have $(a b)^{2} \in\langle a, b\rangle \cap$ $y\left\langle\langle a, b\rangle y^{-1}=v\langle T\rangle v^{-1}\right.$ for $T \subset\{a, b\}$ and $v \in\langle a, b\rangle$. If $T$ is a single element, then $(a b)^{2}$ is conjugate to $a$ or $b$. This is impossible as $(a b)^{2}$ has even length. Hence $T=\langle a, b\rangle$ and so $\langle a, b\rangle=y\langle a, b\rangle y^{-1}$.

Let $\Gamma$ be the Cayley graph of $W$ with respect to $S$. Write $y=x_{1} y_{1} x_{2}$ where $x_{i} \in\langle a, b\rangle$ and $y_{1}$ is the shortest element of the double coset $\langle a, b\rangle y\langle a, b\rangle$. We show that $y_{1}$ commutes with $a$ and $b$. If $\alpha$ is a geodesic in $\Gamma$ from 1 to $y_{1}$, then $a \alpha, b \alpha$, $\alpha a$ and $\alpha b$ are geodesic by the choice of $y_{1}$. Hence by Lemma 9 if $\beta_{1}$ and $\beta_{2}$ are geodesic paths at 1 and $y_{1}$ respectively, in the letters $a, b$, then the paths $\left(\beta_{1}^{-1}, \alpha\right)$ and $\left(\alpha, \beta_{2}\right)$ are geodesic. Now, since $y_{1} a y_{1}^{-1}$ and $y_{1} b y_{1}^{-1}$ are in $\langle a, b\rangle$ they must both be of length 1. I.e. (since $(W, S)$ is even) $y_{1}$ commutes with $a$ and $b$. Furthermore, $y=x_{1} x_{2} y_{1}$. Results in [3] and [2] imply $y_{1}$ is a product of an element of $\langle a, b\rangle$ and an element of $l k_{2}(a, b)$.

Remark 3. For matching edges $[x y]$ and $[a b]$, Theorem 7 concludes that $x y$ or $(x y)^{2}$ is conjugate to $(a b)^{p}$ or $(a b)^{2 p}$. Hence if $c$ is the conjugating element, then $c a b c^{-1}$ commutes with $(x y)^{2}$ and by Proposition 10, $c a b c^{-1}=u v$ for $u \in\langle x, y\rangle$ and $v \in l k_{2}(x, y)$. Similarly, $c^{-1} x y c$ is conjugate to $u^{\prime} v^{\prime}$ for $u^{\prime} \in\langle a, b\rangle$ and $v^{\prime} \in l k_{2}(a, b)$. Hence, certain cases included under Theorem 7 can be improved to say: $(x y)$ is conjugate to $(a b)^{p} t$, where $t$ commutes with $a$ and $b$ and $t^{2}=1$.

Proposition 11. Suppose $(W, S)$ is an even Coxeter system with diagram $V$, $\left(W, S^{\prime}\right)$ is another Coxeter system with diagram $V^{\prime}$, and $[x y]$ and $[y z]$ are distinct edges of $V^{\prime}$ with labels $>2$. If $[a b]$ and $[c d]$ are edges of $V$ which correspond to $[x y]$ and $[y z]$ respectively, then $\{a, b\} \cap\{c, d\}$ contains exactly one element. I.e. The edges $[a b]$ and $[c d]$ share exactly one vertex. 

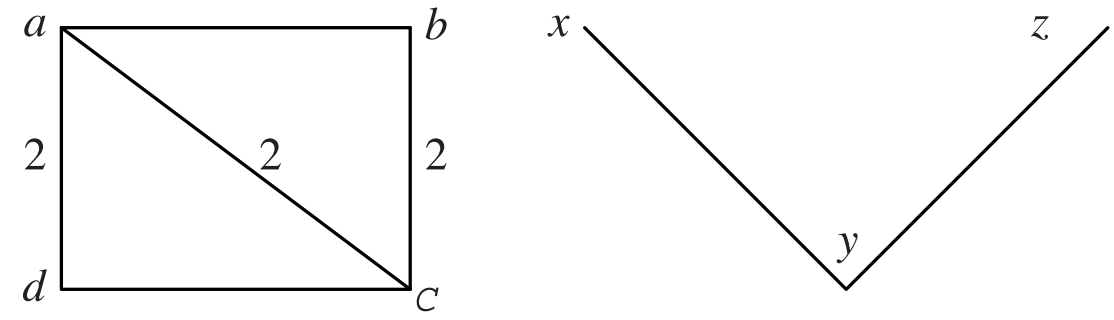

FIGURE 1.

Proof. Suppose that $[x y]$ has label $k>2$ and $[y z]$ has label $m>2$. Observe that $[a b]$ is labeled $l$ where $l=k$ or $l=2 k$ and $[c d]$ by $n$ where $n=m$ or $n=2 m$. The set $\{a, b\} \cap\{c, d\} \neq\{a, b\}$ by the uniqueness of pairing of Theorem 7 . Assume that $\{a, b\} \cap\{c, d\}=\emptyset$. Now $x y$ or $(x y)^{2}$ is equal to $w(a b)^{2 p} w^{-1}$ for some $w \in W$. By conjugation we may assume that either $y z$ or $(y z)^{2}$ is equal to $(c d)^{2 r}$.

Claim 11.1. Not both $a$ and $b$ commute with both $c$ and $d$.

Proof. Otherwise there is a maximal spherical simplex $\sigma$ containing $a, b, c$ and $d$. The group $\langle\sigma\rangle$ is conjugate to $\left\langle\sigma^{\prime}\right\rangle$ for $\sigma^{\prime}$ a simplex of $V^{\prime}$ containing $x, y$ and $z$, which is impossible as $\langle x, y, z\rangle$ is not finite.

Consider the retraction $\alpha: W \rightarrow\langle a, b, c, d\rangle \equiv G$ with kernel $N(S-\{a, b, c, d\})$.

Claim 11.2 Either $a$ or $b$ is an element of $l k_{2}(c, d)$, and either $c$ or $d$ is an element of $l k_{2}(a, b)$.

Proof. Let $\alpha(x) \equiv \bar{x}, \alpha(y) \equiv \bar{y}, \alpha(z) \equiv \bar{z}$ and $\alpha(w) \equiv \bar{w}$. As $(a b)^{2 p}=w^{-1}(x y) w$ or $w^{-1}(x y)^{2} w$, Proposition 10 implies $\bar{w}^{-1} \bar{y} \bar{w}=u_{1} v_{1}$, where $u_{1} \in\langle a, b\rangle$ and $v_{1} \in$ $l k_{2}(a, b)$ (where $l k_{2}$ is taken in $\langle a, b, c, d\rangle$ ), and $\bar{y}=u_{2} v_{2}$, where $u_{2} \in\langle c, d\rangle$ and $v_{2} \in l k_{2}(c, d)$. Note that $v_{1}=c$ or $d$ or 1 and $v_{2}=a$ or $b$ or 1 . If $v_{2}=1$, then $\bar{y} \in\langle c, d\rangle$. The element $\bar{w}^{-1} \bar{y} \bar{w}$ conjugates $(a b)^{2}$ to $(b a)^{2}$. As $\bar{y}(\in\langle c, d\rangle)$ is in the kernel of the retraction of $\langle a, b, c, d\rangle$ to $\langle a, b\rangle$ (with kernel $N(\{c, d\})$ ), this is impossible unless $(a b)^{2}=(b a)^{2}$. If $a b$ has order 4 , then $x y$ has order 4 and Remark 3 implies $x y t=w(a b)^{q} w^{-1}$ where $t$ has order 2 and commutes with $x$ and $y$. In this case we see that $w^{-1} \bar{y} w$ conjugates $a b$ to $b a$. Again this is impossible as $\bar{y} \in\langle c, d\rangle$ is in the kernel of a retraction of $\langle a, b, c, d\rangle$ to $\langle a, b\rangle$ and $a b \neq b a$. Similarly, $v_{1} \neq 1$.

Without loss, we assume that $a \in l k_{2}(c, d)$ and $c \in l k_{2}(a, b)$. (See Figure 1.)

Recall, $T\left(V^{\prime}\right)$ is the product of the edge labels of $V^{\prime}$. Assume that $V^{\prime}$ is a minimal (under $T$ ) counterexample to Proposition 11.

Claim 11.3. There is no edge $[b d]$.

Proof. Otherwise, $[b d]$ has label $>2$ by Claim 11.1. If the edge [st] of $V^{\prime}$ corresponds to $[b d]$ and $s t$ has even label $2 k$, then $[b d]$ has label $2 k$ and $W / N\left((b d)^{2}\right)$ is a smaller counterexample to our proposition. Hence we may assume that $[s t]$ has an odd label. By Proposition $2,\{s, t\} \neq\{x, z\}$, and by Lemma $8, W / N(s t)=W / N\left((b d)^{2}\right)$ is a smaller counterexample.

Claim 11.4 There is no edge $[x z]$. 


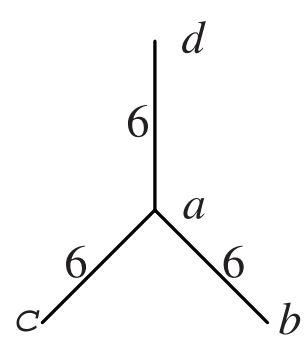

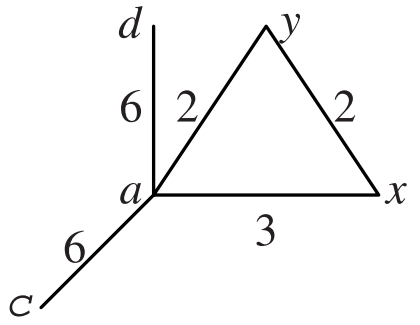

FiguRE 2.

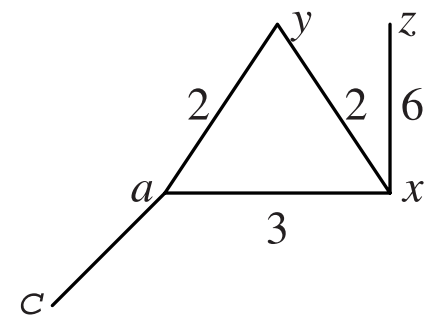

Proof. Otherwise, let $\sigma^{\prime}$ be a maximal simplex of $V^{\prime}$ containing the triangle [xyz]. By [7], there is a simplex $\sigma$ of $V$ such that $\left\langle\sigma^{\prime}\right\rangle$ is conjugate to $\langle\sigma\rangle$. Thus, $\{a, b, c, d\} \subset \sigma$, contradicting Claim 11.3.

Claim 11.5 In $V^{\prime}$, the only edges not labeled 2 are $[x y],[y z]$.

Proof. By the minimality assumption, there are no edges of $V^{\prime}$ with even label $>2$. If $[s t]$ is distinct from $[x y]$ and $[y z]$, and with odd label, then the quotient of $W$ by $N(s t)$ gives a smaller counterexample by Lemma 8

Let $S^{\prime}$ be the vertex set of $V^{\prime}$. Let $\lambda$ be the retraction of $W$ to $\langle x, y, z\rangle$ with kernel $N\left(S^{\prime}-\{x, y, z\}\right)$. Observe that the groups $\langle b, c\rangle,\langle a, d\rangle$, and $\langle a, c\rangle$ inject under $\lambda$, for otherwise $(a b)^{2}$ or $(c d)^{2}$ is in $\operatorname{ker}(\lambda)$ (see Figure 1), which they are not.

Now $\langle x, y, z\rangle=\langle x, y\rangle *\langle y\rangle\langle y, z\rangle$. A maximal simplex $\sigma$ of $V$ containing $\{a, b, c\}$ is such that $\langle\sigma\rangle$ is conjugate to a $\left\langle\sigma^{\prime}\right\rangle$ for $\sigma^{\prime}$ a maximal simplex of $V^{\prime}$. Hence $\lambda(\langle a, b, c\rangle)$ is a subgroup of a conjugate of $\langle x, y\rangle$ or $\langle y, z\rangle$. As $\lambda\left((a b)^{2}\right)$ is in the kernel of the quotient of $\langle x, y, z\rangle$ by $N(x y)$, and $\langle y, z\rangle$ injects under this quotient, $\lambda(\langle a, b, c\rangle)$ is a subgroup of a conjugate of $\langle x, y\rangle$. Similarly, $\lambda(\langle a, c, d\rangle)$ is a subgroup of a conjugate of $\langle y, z\rangle$. Hence (simply consider edge and vertex stabilizers of the Bass-Serre tree for $\langle x, y\rangle *\langle y\rangle\langle y, z\rangle) \lambda(\langle a, c\rangle)$ is a subgroup of a conjugate of $\langle y\rangle$. But this is impossible as $\langle y\rangle$ has order 2. This finishes the proof of Proposition 11

Example 1. Three diagrams for an even Coxeter group are shown in Figure 2. Isomorphisms between presentations determined by these diagrams are given by: $a \rightarrow a, c \rightarrow c, d \rightarrow d, x \rightarrow b a b$ and $y \rightarrow(a b)^{3}$ (a triangle/edge exchange) and $a \rightarrow a, c \rightarrow c, x \rightarrow x, y \rightarrow y$ and $z \rightarrow \operatorname{axadaxa}$ (a twist of $\{d\}$ around $[a x]$ ). The correspondence of Proposition 7 between edges of the first and last diagrams of Figure 2 match the adjacent edges $[a c]$ and $[a d]$ with the non-adjacent edges $[a c]$ and $[x z]$, respectively.

Proposition 12. Suppose $(W, S)$ is an even Coxeter system with diagram $V$, $\left(W, S^{\prime}\right)$ is another Coxeter system with diagram $V^{\prime}$, and $[a b]$ and $[a c]$ are distinct edges of $V$ with labels $>2$. If $[x y]$ and $[z v]$ are edges of $V^{\prime}$ which correspond to $[a b]$ and $[a c]$ respectively, then either there is an odd edge path in $V^{\prime}$ connecting a vertex of $\{x, y\}$ and a vertex of $\{z, v\}$, or $\{x, y\} \cap\{z, v\}$ contains exactly one element.

Proof. Suppose there is no odd edge path in $V^{\prime}$ with one vertex in $\{x, y\}$ and one vertex in $\{z, v\},\{x, y\} \cap\{z, v\}=\emptyset$, and $V^{\prime}$ is a minimal (over $T\left(V^{\prime}\right)$ ) such counterexample to our proposition. 

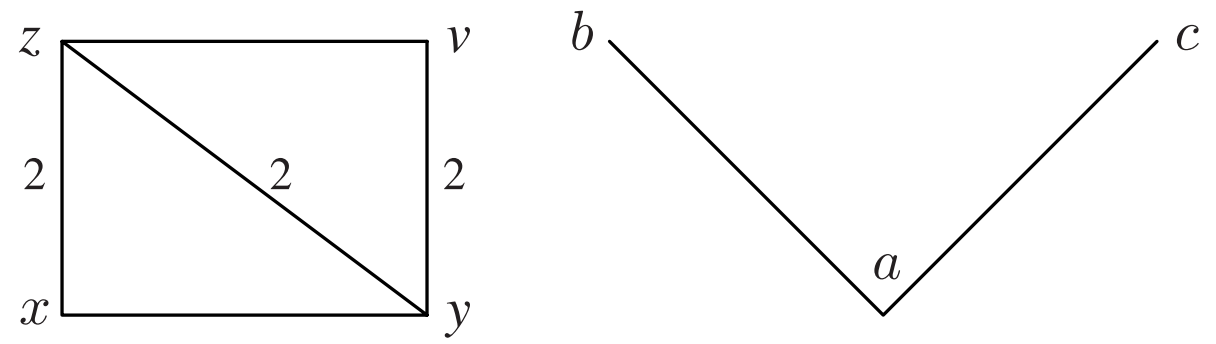

FIGURE 3.

Claim 12.1 The only edges of $V^{\prime}$ not labeled 2 are $[x y]$ and $[z v]$.

Proof. By the minimality of $T\left(V^{\prime}\right)$, there is no edge of $V^{\prime}$ with a label that is even and $>2$, other than possibly $[x y]$ and $[z v]$. Also by the minimality of $T\left(V^{\prime}\right)$ and Lemma 8 there is no edge of $V^{\prime}$ with an odd label other than $[x y]$ or $[z v]$.

By Claim 12.1 and Theorem 7 , the only edges of $V$ not labeled 2 are $[a b]$ and $[a c]$. Let $\sigma$ be a maximal spherical simplex of $V$ containing $\{a, b\}$ and $\sigma^{\prime}$ be a maximal spherical simplex in $V^{\prime}$ such that $\bar{w}\left\langle\sigma^{\prime}\right\rangle \bar{w}^{-1}=\langle\sigma\rangle$ for some $\bar{w} \in W$. Then $\{x, y\} \subset \sigma^{\prime}$. If neither $z$ nor $v$ is an element of $\sigma^{\prime}$, then $W / N(\sigma)=W / N\left(\sigma^{\prime}\right)$ is a Coxeter group with a diagram obtained from $V$ (respectively $V^{\prime}$ ) by removing the vertices of $\sigma$ (respectively $\sigma^{\prime}$ ). But one of these diagrams has all edges labeled 2 and the other has an edge with label $>2$, which is impossible. We conclude that $v$ or $z$ is an element of $\sigma^{\prime}$, and so $v$ or $z$ is an element of $l k_{2}(x, y)$. Similarly, $x$ or $y$ is an element of $l k_{2}(z, v)$. Say $z \in l k_{2}(x, y)$ and $y \in l k_{2}(z, v)$. See Figure 3.

Consider the retraction $\tau$ of $W$ to $\langle a, b, c\rangle$ with kernel $N(S-\{a, b, c\})$. By Theorem $7\left\{\left\{(x y)^{2},(z v)^{2}\right\} \cap \operatorname{ker}(\tau)=\emptyset\right.$. Hence $\{x, y, z, v, z y, x z, y v\} \cap \operatorname{ker}(\tau)=\emptyset$ (see Figure 3). There is no edge $[b c]$, as every simplex of $V^{\prime}$ (and hence every simplex of $V)$ is spherical. Observe that $\langle a, b, c\rangle=\langle a, b\rangle *\langle a\rangle\langle a, c\rangle$. Note that $\tau(x y)$ has order $>2$, and is an element of a conjugate of $\langle a, b\rangle$. Hence $\tau(x y)$ cannot be an element of distinct conjugates of $\langle a, b\rangle$, or some conjugate of $\langle a, c\rangle$, since otherwise (simply consider vertex and edge stabilizers of the Bass-Serre tree for $\langle a, b\rangle *\langle a\rangle\langle a, c\rangle$ ) $\tau(x y)$ is an element of a conjugate of $\langle a\rangle$, an order 2 group. Therefore, $\tau(\langle x, y, z\rangle)$ is a subgroup of a conjugate of $\langle a, b\rangle$ and $\tau(\langle y, z, v\rangle)$ is a subgroup of a conjugate of $\langle a, c\rangle$. But this implies (again consider vertex and edge stabilizers of the Bass-Serre tree for $\langle a, b\rangle *\langle a\rangle\langle a, c\rangle)$ that $\alpha(\langle z, y\rangle)$ is a subgroup of a conjugate of $\langle a\rangle$. This is impossible, and the proof of the proposition is complete.

The following result is used in [1, so we cannot use [1] to simplify the proof.

Lemma 13. Suppose $V$ is an even diagram for the finitely generated Coxeter group $W$ and $V^{\prime}$ is another diagram for $W$. If $[a b c]$ is a triangle of $V$ having at least two edges with label $>2$, then the edges of $[a b c]$ with label $>2$ correspond to edges with label $>2$ of a triangle $[x y z]$ of $V^{\prime}$.

Proof. Let $\sigma$ be a maximal simplex of $V$ containing [abc]. By [7, there is a maximal simplex $\sigma^{\prime}$ in $V^{\prime}$ such that $\langle\sigma\rangle$ is conjugate to $\left\langle\sigma^{\prime}\right\rangle$. By Theorem 7 applied to $\sigma$ and $\sigma^{\prime}$ and the uniqueness conclusion of Theorem $\left[7\right.$ applied to $V$ and $V^{\prime}, \sigma^{\prime}$ contains the edges of $V^{\prime}$ corresponding to those of $[a b c]$ that have label $>2$. First we show the edges of $\sigma^{\prime}$ with label $>2$ and corresponding to those of $[a b c]$ with label $>2$ 
are mutually adjacent. If not, say $[x y]$ and $[z v]$ are two such non-adjacent edges. By Proposition 12 (applied to the even Coxeter group $\langle\sigma\rangle$ ) there is an odd labeled edge (in $\sigma^{\prime}$ ) adjacent to $[x y]$, but this is impossible by Proposition 2 .

If an edge of $[a b c]$ is labeled 2, we are finished. Otherwise, the edges of $\left\langle\sigma^{\prime}\right\rangle$ corresponding to those of $[a b c]$ must have even labels by Proposition 2 and either form a triangle or triad. If a triad is formed, then we may assume that $[a b]$ corresponds to $[x y]$ in $V^{\prime},[b c]$ corresponds to $[x z]$ and $[a c]$ corresponds to $[x v]$. Now assume that $V$ is a minimal (with respect to $T(V)$ ) counterexample to the lemma. Then $V=\sigma$ and $V^{\prime}=\sigma^{\prime}$. By minimality, every edge of $V$ except $[a b],[b c]$ and $[a c]$ has label 2. Similarly for $V^{\prime}$. In particular, $V$ and $V^{\prime}$ are even. By conjugation, we may assume that $\left\langle(x y)^{2}\right\rangle=\left\langle(a b)^{2}\right\rangle$. As $x$ conjugates $(a b)^{2}$ to $(b a)^{2}$, Proposition 10 implies that $x=u_{1} t_{1}$, where $u_{1} \in\langle a, b\rangle$ and $t_{1} \in l k_{2}(a, b)$. Similarly, $x=w_{2} u_{2} t_{2} w_{2}^{-1}$ where $w_{2} \in W, u_{2} \in\langle b, c\rangle$ and $t_{2} \in l k_{2}(b, c)$ and $x=w_{3} u_{3} t_{3} w_{3}^{-1}$ where $w_{3} \in W$, $u_{3} \in\langle a, c\rangle$ and $t_{3} \in l k_{2}(a, c)$. Now

$$
\begin{gathered}
x \in\left(\left\langle\{a, b\} \cup l k_{2}(a, b)\right\rangle\right) \cap\left(w_{2}\left\langle\{b, c\} \cup l k_{2}(b, c)\right\rangle w_{2}^{-1}\right) \\
\cap\left(w_{3}\left\langle\{a, c\} \cup l k_{2}(a, c)\right\rangle w_{3}^{-1}\right)=v\langle T\rangle v^{-1}
\end{gathered}
$$

where $T \subset\{a, b\} \cup l k_{2}(a, b)$. Clearly, $c \notin T$. As no conjugate of $a$ is an element of $w_{2}\left\langle\{b, c\} \cup l k_{2}(b, c)\right\rangle w_{2}^{-1}, a \notin T$. Similarly, $b \notin T$. But then $T$ is central in $W$, implying $x$ is central, the desired contradiction.

Lemma 14. Suppose $V$ is an even diagram for the finitely generated Coxeter group $W$ and $V^{\prime}$ is another diagram for $W$. If $[x y z]$ is a triangle of $V^{\prime}$ having at least two edges with label $>2$, then the edges of $[x y z]$ with label $>2$ correspond to edges with label $>2$ of a triangle $[a b c]$ of $V$.

Proof. By Proposition 2 each edge of $[x y z]$ has an even label. Suppose $V^{\prime}$ is a minimal (with respect to $T\left(V^{\prime}\right)$ ) counterexample. By Lemma 8 , $V^{\prime}$ contains no odd labeled edge, and so $V^{\prime}$ is even. Now apply Lemma 13 .

Remark 4. Observe in each of the last two lemmas that if we begin with a triangle with exactly one edge labeled 2 , we do not conclude that the corresponding triangle has an edge labeled 2. This can now be resolved by combining the last two lemmas. More specifically, we see that if $W$ is an even Coxeter group, $V$ and $V^{\prime}$ are diagrams for $W$ and $[a b c]$ is a triangle of $V$ with only one edge with label 2, then there is a corresponding triangle $[x y z]$ of $V^{\prime}$ with only one edge labeled 2. If $[a b c]$ has no edge labeled 2, then $[x y z]$ has no edge labeled 2 .

As a direct application of Lemma 13 and Lemma 14 we have an analogue for Proposition 2 ,

Lemma 15. Suppose $W$ is an even Coxeter group, $V$ and $V^{\prime}$ are diagrams for $W$, $[x y]$ is an odd labeled edge of $V^{\prime}$ and $[a b]$ the edge of $V$ corresponding to $[x y]$. Then any triangle containing $[a b]$ has two edges labeled 2.

Proposition 16. Suppose $V$ is an even diagram for the finitely generated Coxeter group $W$ and $V^{\prime}$ is another diagram for $W$. If $[x y]$ is an odd labeled edge of $V^{\prime}$ and $[a b]$ is the corresponding edge of $V$, then with the exception of [ab], every edge adjacent to $a$ is labeled 2 or every edge adjacent to $b$ is labeled 2. 
Proof. Suppose $[x y]$ has label $2 k+1$. It suffices to show:

Claim 16.1 There is no edge path $([c a],[a b],[b d])$ in $V$ such that each edge has label $>2$.

Proof. Since $[x y]$ has an odd label, Lemma 15] implies that the path $([c a],[a b],[b d])$ does not form a triangle. Suppose that $[c a]$ and $[b d]$ correspond to $[u v]$ and $[s t]$ respectively in $V^{\prime}$. By Proposition [12, select a shortest path with odd labeled edges, $e_{1}, \ldots, e_{n}$ from $\{u, v\}$ to $\{x, y\}$. Assume that $e_{i}=\left[x_{i-1} x_{i}\right]$ for all $i$. If $e_{j}=[s t]$, then a diagram $\bar{V}^{\prime}$ for $W / N\left(\left\{x_{0} x_{1}, \ldots, x_{j-2} x_{j-1}\right\}\right)$ is obtained from $V^{\prime}$ by collapsing each edge of the set $\left\{e_{1}, \ldots, e_{j-1}\right\}$. The corresponding diagram $\bar{V}$ for $W / N\left(\left\{x_{0} x_{1}, \ldots, x_{j-2} x_{j-1}\right\}\right)$ is obtained from $V$ by replacing each label of an edge corresponding to one of $\left\{e_{1}, \ldots, e_{j-1}\right\}$ by 2 . In $\bar{V}^{\prime}$, [uv] and $[s t]$ are adjacent, but $[c a]$ and $[b d]$ are not, contradicting Proposition [11. We conclude that $[s t]$ is not in $\left\{e_{1}, \ldots, e_{n}\right\}$. Similarly, if $d_{1}, \ldots, d_{m}$ is a shortest odd labeled edge path from $\{x, y\}$ to $\{s, t\}$, we may assume that $[u v]$ is not in $\left\{d_{1}, \ldots, d_{m}\right\}$. Assume that $d_{i}=\left[y_{i-1} y_{i}\right]$ for all $i$. A diagram $\tilde{V}^{\prime}$ is obtained for the group $\tilde{W} \equiv W / N\left(\left\{x_{0} x_{1}, \ldots, x_{n-1} x_{n}, x y, y_{0} y_{1}, \ldots, y_{m-1} y_{m}\right\}\right)$ by collapsing the edges $e_{1}, \ldots, e_{n},[x y], d_{1}, \ldots, d_{m}$ of $V^{\prime}$. Hence in $\tilde{V}^{\prime}$, [uv] and $[s t]$ are adjacent. Let $\tilde{V}$ be the diagram for $\tilde{W}$ obtained from $V$ by changing the edge labels of the edges of $V$ corresponding to $e_{1}, \ldots, e_{n},[x y], d_{1}, \ldots, d_{m}$ to 2 . In $\tilde{V},[c a]$ and $[b d]$ are not adjacent, contradicting Proposition 11

The following theorem classifies even rigid Coxeter groups.

Theorem 17. If $V$ is an even diagram for the Coxeter group $W$, then $W$ has a diagram that is not even if and only if there is an edge $[a b]$ in $V$ with label $2(2 k+1)$ for $k>0$, such that with the exception of [ab], every edge of $V$ containing a is labeled 2 and if $[a c]$ is such an edge, then there is an edge $[b c]$ with label 2.

Proof. If $[a b]$ is an edge as described in the theorem, then $\left\langle a, b: a^{2}=b^{2}=\right.$ $\left.(a b)^{2(2 k+1)}=1\right\rangle$ is isomorphic to the group $\left\langle x, y, z: x^{2}=y^{2}=z^{2}=(x z)^{2}=\right.$ $\left.(y z)^{2}=(z y)^{2 k+1}=1\right\rangle$ by the map extending $x \rightarrow a, y \rightarrow b a b$ and $z \rightarrow(a b)^{2 k+1}$. It is elementary to see that the edge $[a b]$ in $V$ can be replaced by the triangle $[x y z]$ to give a new diagram for $W$.

The proof of the converse is more delicate. Recall that $T(V)$ is the product of all edge labels in $V$. We assume from this point on that $V$ is a minimal (with respect to $T$ ) counterexample to our theorem. Let $V^{\prime}$ be a diagram for $W$ with odd labeled edge $[x y]$. Assume that $[x y]$ corresponds to the edge $[a b]$ of $V$.

Claim 17.1 With the exception of [ab] every edge of $V$ is labeled 2 (and hence [xy] is the only edge of $V^{\prime}$ not labeled 2).

Proof. If $[c d]$ is an edge labeled $n>2$ and $\{c, d\} \cap\{a, b\}=\emptyset$, then the quotient of $W$ by $N\left((c d)^{2}\right)$ is a "smaller" counterexample. If $[a c]$ has label $n>2$, then there is no edge [bc] by Lemma [15. Again the quotient of $W$ by $N\left((a c)^{2}\right)$ is a smaller counterexample. Similarly, there is no edge $[b c]$ with label $>2$.

Claim 17.2 Suppose $\sigma$ and $\sigma^{\prime}$ are simplices of $V$ and $V^{\prime}$ respectively such that $\langle\sigma\rangle$ is conjugate to $\left\langle\sigma^{\prime}\right\rangle$. Then $\sigma$ contains $\{a, b\}$ if and only if $\sigma^{\prime}$ contains $\{x, y\}$. Also, $\sigma$ contains exactly one element of $\{a, b\}$ if and only if $\sigma^{\prime}$ contains exactly one element of $\{x, y\}$. 
Proof. The group $\langle\sigma\rangle$ (respectively $\left\langle\sigma^{\prime}\right\rangle$ ) is non-abelian if and only if $\{a, b\} \subset \sigma$ (respectively $\{x, y\} \subset \sigma^{\prime}$ ). Hence the first conclusion of the lemma follows.

The group $W / N(\sigma)$ (respectively $W / N\left(\sigma^{\prime}\right)$ ) is abelian iff $a$ or $b \in \sigma$ (respectively $x$ or $\left.y \in \sigma^{\prime}\right)$. But $W / N(\sigma)=W / N\left(\sigma^{\prime}\right)$.

To finish Theorem [17, it suffices to show that $V$ cannot contain edges $[a d]$ and $[b c]$ $(d \neq b$ and $c \neq a)$ such that there is no edge between $b$ and $d$ and no edge between $a$ and $c$. (There may or may not be an edge $[c d]$.) Assume otherwise. Let $\sigma(a, d)$ and $\sigma(a, b)$ be maximal simplices of $V$ containing $\{a, d\}$ and $\{a, b\}$ respectively. Let $\sigma=\sigma(a, d) \cap \sigma(a, b)$. Note that $a \in \sigma$, but $\{b, c, d\} \cap \sigma=\emptyset$. By conjugation we may assume that $\sigma^{\prime}(a, b)$ is a maximal simplex of $V^{\prime}$ such that $\left\langle\sigma^{\prime}(a, b)\right\rangle=\langle\sigma(a, b)\rangle$ and that $w\left\langle\sigma^{\prime}(a, d)\right\rangle w^{-1}=\langle\sigma(a, d)\rangle$ for $\sigma^{\prime}(a, d)$ a maximal simplex of $V^{\prime}$ and $w \in W$. Then $\langle\sigma\rangle=\left\langle\sigma^{\prime}(a, b)\right\rangle \cap w\left\langle\sigma^{\prime}(a, d)\right\rangle w^{-1}=v\langle T\rangle v^{-1}$, for some $v \in\left\langle\sigma^{\prime}(a, b)\right\rangle$, and $T \subset \sigma^{\prime}(a, b)$. By Claim 17.2 either $x$ or $y$, but not both, is an element of $T$.

Let $q$ be the retraction of $W$ to $\langle a, b, c, d\rangle$ with kernel $N(S-\{a, b, c, d\})$. Then, $q(\langle a\rangle)=q(\langle\sigma(a, b)\rangle \cap\langle\sigma(a, d)\rangle)=q\left(v\langle T\rangle v^{-1}\right)$. Observe that $x$ is conjugate to $y$, $\langle x y\rangle$ is conjugate to $\left\langle(a b)^{2}\right\rangle$ and $q(a b)$ has order $2(2 k+1)$. Thus, $q(x) \neq 1 \neq q(y)$ and so $q(\langle a\rangle)$ is conjugate to $q(\langle x\rangle)$ and $q(\langle y\rangle)$. Hence $q(a)$ is conjugate to $q(x)$ and $q(y)$. Similarly for $b$. This implies $q(a)$ and $q(b)$ are conjugate, the desired contradiction. Theorem 17 is finished.

\section{The proof of Proposition 3}

It remains to prove Propositions 3 and 4 . In this section we complete the former.

Proof of Proposition 3. Suppose $V$ is an even diagram for the Coxeter group $W$, $[x y]$ is an odd edge in a diagram $V^{\prime}$ for $W$ and every edge $[x c]$ of $V^{\prime}$ for $c \neq y$ is such that $c \in l k_{2}(x, y)$. We wish to show that there exists of a vertex $u \in l k_{2}(x, y)$ such that if $[u c]$ is an edge with $c \notin\{x, y\}$, then $[u c]$ has label $2, c \in l k_{2}(x, y)$, and any simplex $\sigma^{\prime}$ of $V^{\prime}$ that contains $u$ (respectively $x$ and $y$ ) and is such that $\left\langle\sigma^{\prime}\right\rangle$ is conjugate to $\langle\sigma\rangle$ for $\sigma$ a simplex of $V$, contains $\{x, y\}$ (respectively $u$ ).

Lemma 18. Suppose $(W, S)$ is an even Coxeter system with diagram $V, V^{\prime}$ is another diagram for $W$, and $\left\langle\sigma^{\prime}\right\rangle=w\langle\sigma\rangle w$ for $\sigma^{\prime}$ a simplex of $V^{\prime}, \sigma$ a simplex of $V$ and $w \in W$. If $f:\left\langle\sigma^{\prime}\right\rangle \rightarrow \mathbb{Z}_{2} \equiv\{-1,1\}$ is a homomorphism, let $N$ be the normal closure in $W$ of ker $(f)$. Then $W / N$ is an even Coxeter group with diagram obtained from $V$ by removing the vertices of $\sigma_{1} \equiv\left\{s \in \sigma: f\left(w s w^{-1}\right)=1\right\}$ and identifying the vertices of $\sigma-\sigma_{1}$.

Proof. The kernel of $f$ is generated by $K^{\prime}$, the normal closure in $\left\langle\sigma^{\prime}\right\rangle$ of $\left\{s \in \sigma^{\prime}\right.$ : $f(s)=1\} \cup\left\{s t: s, t \in \sigma^{\prime}, f(s)=f(t) \neq 1\right\}$. Hence $N$ is the normal closure of $K^{\prime}$ in $W$.

As $\left\langle\sigma^{\prime}\right\rangle=w\langle\sigma\rangle w^{-1}, K^{\prime}$ can also be described as the normal closure in $w\langle\sigma\rangle w^{-1}$ of $K=\left\{w s w^{-1}: s \in \sigma\right.$ and $\left.f\left(w s w^{-1}\right)=1\right\} \cup\left\{w s t w^{-1}: s, t \in \sigma\right.$ and $f\left(w s w^{-1}\right)=$ $\left.f\left(w t w^{-1}\right) \neq 1\right\}$. Now, in $W$, the normal closure of $K^{\prime}, K$ and $w^{-1} K w$ are the same.

Lemma 19. Suppose $(W, S)$ is an even Coxeter system with diagram $V$ and $V^{\prime}$ is another diagram for $W$ with odd edge $[x y]$. There exists a vertex $u \in V^{\prime}-\{x, y\}$ such that $u$ is contained in the intersection of all simplicies $\sigma^{\prime}$ containing $\{x, y\}$ and such that $\left\langle\sigma^{\prime}\right\rangle$ is conjugate to $\langle\sigma\rangle$ for $\sigma$ a simplex of $V$. Furthermore if $\sigma^{\prime}$ is a 
simplex of $V^{\prime}$ containing $u$ and such that $\left\langle\sigma^{\prime}\right\rangle$ is conjugate to $\langle\sigma\rangle$ for $\sigma$ a simplex of $V$, then $\{x, y\} \subset \sigma^{\prime}$.

Proof. Assume $V^{\prime}$ is a minimal (with respect to $T\left(V^{\prime}\right)$ ) counterexample. Let $\delta^{\prime}$ be the intersection of all simplices $\sigma^{\prime}$ of $V^{\prime}$, containing $\{x, y\}$ and such that $\left\langle\sigma^{\prime}\right\rangle$ is conjugate to $\langle\sigma\rangle$ for some simplex $\sigma$ of $V$. As $\langle x, y\rangle$ is not an even Coxeter group, $\delta^{\prime} \neq\{x, y\}$. If $u \in \delta^{\prime}-\{x, y\}$, there is no odd path in $V^{\prime}$ from $u$ to $x$ or $y$, by Proposition 2 and Lemma 8 . For each $u \in \delta^{\prime}-\{x, y\}$ assume there is a simplex $\beta^{\prime}$ of $V^{\prime}$ such that $\left\langle\beta^{\prime}\right\rangle$ is conjugate to $\langle\beta\rangle$ for $\beta$ a simplex of $V$ and $u \in \beta^{\prime}$, but $\{x, y\} \not \subset \beta^{\prime}$. By intersecting, we may assume that each such $\beta^{\prime} \subset \delta^{\prime}$. Select one such $\beta^{\prime}$. By Lemma 18, the map of $\left\langle\beta^{\prime}\right\rangle$ to $\mathbb{Z}_{2}$ that sends $\beta^{\prime}-\{x, y\}$ to 1 and $\beta^{\prime} \cap\{x, y\}$ to -1 defines a smaller counterexample.

Let $\delta^{\prime}$ be the intersection of all simplices $\sigma^{\prime}$ of $V^{\prime}$ such that $\{x, y\} \subset \sigma^{\prime}$ and $\left\langle\sigma^{\prime}\right\rangle$ is conjugate to $\langle\sigma\rangle$ for some simplex $\sigma$ of $V$. By Lemma 19, $\delta^{\prime}$ contains a vertex $v$ such that if $v \in \sigma^{\prime}$, where $\sigma^{\prime}$ is a simplex of $V^{\prime}$ and $\left\langle\sigma^{\prime}\right\rangle$ is conjugate to $\langle\sigma\rangle$ for some simplex $\sigma$ of $V$, then $\{x, y\} \subset \sigma^{\prime}$. We call such a $\delta^{\prime}$-vertex $\{x, y\}$-linked or simply linked.

It suffices to show that $\delta^{\prime}$ contains a linked vertex $v^{\prime}$, such that every edge of $V^{\prime}$ containing $v^{\prime}$ is labeled 2. Otherwise, assume that $V^{\prime}$ is a minimal counterexample. Then each linked vertex belongs to an edge with label $>2$.

Suppose $[s t]$ is an edge of $V^{\prime}$ with label $>2$ and neither $s$ nor $t$ is linked. If $[s t]$ has an even (respectively odd) label, then the even Coxeter group $W / N\left((s t)^{2}\right)$ (respectively $W / N(s t)$ ), with diagram $\bar{V}^{\prime}$, obtained from $V^{\prime}$ by changing the label of $[s t]$ to a 2 (respectively identifying $s$ and $t$ ), is a smaller counterexample. (Note that if a vertex is not $\{x, y\}$-linked in $V^{\prime}$, then it is not $\{x, y\}$-linked in $\bar{V}^{\prime}$, and an $\{x, y\}$-linked vertex of $V^{\prime}$ may not be $\{x, y\}$-linked in $\bar{V}^{\prime}$.) Hence, every edge with label $>2$ (other than $[x y]$ ) contains a linked vertex.

Now $\left\langle\delta^{\prime}\right\rangle$ is conjugate to $\langle\delta\rangle$ for some simplex $\delta$ of $V$. Then $\delta^{\prime}$ contains more vertices than $\delta$. (If the odd edges of $\delta^{\prime}$ are collapsed to single vertices and each even $>2$ label of $V^{\prime}$ is changed to 2, we obtain the (unique) diagram for a right angled (all edge labels are 2) Coxeter group. The diagram for this group is also obtained from $\delta$ if each even $>2$ label of $\delta$ is changed to 2. This latter description of this diagram has the same number of vertices as $\delta$, but the former diagram has fewer vertices (from the collapse of $[x y]$ ) than $\delta^{\prime}$.)

We obtain the desired contradiction by showing $\delta$ has at least as many vertices as $\delta^{\prime}$. Let $A^{\prime}(A)$ be the set of vertices of $\delta^{\prime}(\delta)$ that belong to an edge of $V^{\prime}(V)$ with label $>2$. As $\left\langle\delta^{\prime}\right\rangle$ is finite, no two adjacent edges of $\delta^{\prime}$ have labels $>2$. Similarly for $\delta$. The matching of Theorem 7 for $\delta^{\prime}$ and $\delta$ respects the matching for $V^{\prime}$ and $V$. Hence $\delta^{\prime}$ contains an edge with label $>2$ iff $\delta$ contains the matching edge. So the number of vertices of $A^{\prime}$ that belong to an edge of $\delta^{\prime}$ with label $>2$ agrees with the number of vertices of $A$ that belong to an edge of $\delta$ with label $>2$.

Suppose $[s t]$ is an edge of $V^{\prime}$ with label $>2$ and $s \in \delta^{\prime}, t \notin \delta^{\prime}$, and $s$ is not a vertex of an edge in $\delta^{\prime}$ with label $>2$. Suppose $[a b]$ is the edge of $V$ matching $[s t]$. Then $\{a, b\} \not \subset \delta$. Considering the quotient of $W$ by $N(\delta)=N\left(\delta^{\prime}\right)$, we see $\{a, b\} \cap \delta \neq \emptyset$. Hence we assume $b \in \delta$ and $a \notin \delta$. We wish to see that $b$ does not belong to an edge of $\delta$ with label $>2$. Suppose $[b c]$ is such an edge and $[u v]$ is an edge of $\delta^{\prime}$ matching [bc]. By Proposition 12 there is an odd edge path from [st] to $[u v]$. The first edge of this path cannot be $[t p]$, since then $p \in \delta^{\prime}$ and Proposition 2 is violated. Hence the first edge must be [sp] and by assumption, $p \notin \delta^{\prime}$, so 
$p \notin\{u, v\}$. If $[p q]$ is the next edge, then $q \in \delta^{\prime}$ and again Proposition 2 is violated. We conclude that $b$ does not belong to an edge of $\delta$ with label $>2$.

Recall from Proposition 11 that if edges with label $>2$ of $V^{\prime}$ are adjacent, then their matching edges in $V$ are adjacent. We show that $\left|A^{\prime}\right|=|A|$ by verifying the following three statements.

First, if $[s t]$ and $[u v]$ are (non-adjacent) edges with labels $>2$ of $V^{\prime}$ such that $\{s, u\} \subset \delta^{\prime}, t, v \notin \delta^{\prime}$, and neither $s$ nor $u$ belongs to an edge of $\delta^{\prime}$ with label $>2$, then the corresponding edges of $V$, call them $[a b]$ and $[c d]$ respectively, are not adjacent. Otherwise, there is an odd edge path from $[s t]$ to $[u v]$. A contradiction is obtained as in the former argument.

Suppose [st] and $[s p]$ are edges of $V^{\prime}$ with labels $>2, s \in \delta^{\prime},\{t, p\} \subset V^{\prime}-\delta^{\prime}$ and $s$ not a vertex of an edge of $\delta^{\prime}$ with label $>2$. Then if $[a b]$ and $[a c]$ are the edges of $V$ corresponding to [st] and [sp] respectively, either $a \in \delta$ and $a$ is not adjacent to an edge of $\delta$ with label $>2$ or $\{b, c\} \subset \delta$ and neither $b$ nor $c$ is adjacent to an edge of $\delta$ with label $>2$. We show the latter scenario cannot occur. Otherwise, the triangle $[a b c]$ is contained in a maximal simplex $\tau$ of $V$, and $\langle\tau\rangle$ is conjugate to $\left\langle\tau^{\prime}\right\rangle$ for $\tau^{\prime}$ a maximal simplex of $V^{\prime}$. Hence $\{s, t, p\}$ forms a triangle, no edge of which has an odd label. By Proposition 2 and Lemma 8 , there is no odd edge path from $\{t, p\}$ to $\delta^{\prime}$ and no odd edge path between $t$ and $p$. Let $K$ be the kernel of the map of $\left\langle\sigma^{\prime}\right\rangle$ to $\mathbb{Z}_{2}$ that takes $\sigma^{\prime}-\{s\}$ to 1 and $s$ to -1 . A diagram for $\bar{W} \equiv W / N(K)$ is obtained from $V^{\prime}$ by removing the vertices $\sigma^{\prime}-\{s\}$ and all vertices that can be connected to $\sigma^{\prime}-\{s\}$ by an odd labeled edge path. The subgroup $\langle s, t, p\rangle$ of $W$ injects under this quotient map. By Lemma 18, a diagram for $W / N(K)$ is obtained from $V$ by removing some vertices of $\delta$ and identifying all others. Neither $b$ nor $c$ is removed since Theorem 7 (applied to $W / N(K)$, and the two diagrams for this group) implies that the edges $[s t]$ and $[s p]$ correspond to $[a b]$ and $[a c]$ respectively. Similarly $b$ and $c$ are not identified.

Finally, suppose $[s t]$ and $[s p]$ are edges of $V^{\prime}$ with labels $>2,\{t, p\} \subset \delta^{\prime}, s \in$ $V^{\prime}-\delta^{\prime}$ and neither $t$ nor $p$ is a vertex of an edge of $\delta^{\prime}$ with label $>2$. Then if $[a b]$ and $[a c]$ are the edges of $V$ corresponding to [st] and $[s p]$ respectively, either $a \in \delta$ and $a$ is not adjacent to an edge of $\delta$ with label $>2$ or $\{b, c\} \subset \delta$ and neither $b$ nor $c$ is adjacent to an edge of $\delta$ with label $>2$. We show the former scenario cannot occur. As $\{s, t, p\}$ forms a triangle, no edge of this triangle has an odd label. If each odd labeled edge of $V^{\prime}$ is identified to a vertex, then the resulting diagram is even with Coxeter group $\bar{W}$ a quotient of $W$. Another diagram for $\bar{W}$ is obtained from $V$ by changing labels on edges corresponding to odd labeled edges to 2 . The triangles $[s t p]$ and $[a b c]$ induce triangles in the respective diagrams for $\bar{W}$, and the conjugate simplices groups $\left\langle\sigma^{\prime}\right\rangle$ and $\langle\sigma\rangle$ induce conjugate simplex groups in $\bar{W}$. Since both of these diagrams are even, the previous argument shows this is impossible.

By a completely analogous argument, we have:

Claim 3.1 If $\sigma^{\prime} \subset \delta^{\prime}$ is such that $\left\langle\sigma^{\prime}\right\rangle$ is conjugate to $\langle\sigma\rangle$ for $\sigma \subset \delta$, then $\left|A^{\prime} \cap \sigma^{\prime}\right|=$ $|A \cap \sigma|$.

Let $B^{\prime}(B)$ be the vertices of $\delta^{\prime}(\delta)$ that do not belong to an edge with label $>2$. So $\delta^{\prime}-A^{\prime}=B^{\prime}$ and $\delta-A=B$. It suffices to show $\left|B^{\prime}\right| \leq|B|$. If $b^{\prime} \in B^{\prime}$, then there exists a simplex $\sigma_{b^{\prime}}^{\prime} \subset \delta^{\prime}$ such that $b^{\prime} \in \sigma_{b^{\prime}}^{\prime},\left\langle\sigma_{b^{\prime}}^{\prime}\right\rangle$ is conjugate to $\left\langle\sigma_{b^{\prime}}\right\rangle$ for some simplex $\sigma_{b^{\prime}} \subset \delta$ and not both $x$ and $y$ are in $\sigma_{b^{\prime}}^{\prime}$. Since $\sigma_{b^{\prime}}^{\prime}$ can contain no linked vertex, it must be right angled and so $\left|\sigma_{b^{\prime}}^{\prime}\right|=\left|\sigma_{b^{\prime}}\right|$. By Claim 3.1, $\left|\sigma_{b^{\prime}}^{\prime} \cap A^{\prime}\right|=\left|\sigma_{b^{\prime}} \cap A\right|$, so $\left|\sigma_{b^{\prime}}^{\prime} \cap B^{\prime}\right|=\left|\sigma_{b^{\prime}} \cap B\right|$. 
Claim 3.2 If $\sigma_{1}^{\prime}, \ldots, \sigma_{n}^{\prime}$ are subsets of $\delta^{\prime}$, and $\left\langle\sigma_{i}^{\prime}\right\rangle$ is right angled and conjugate to $\left\langle\sigma_{i}\right\rangle$ for $\sigma_{i} \subset \delta$, then $\left|B^{\prime} \cap\left(\bigcap_{i=1}^{n} \sigma_{i}^{\prime}\right)\right|=\left|B \cap\left(\bigcap_{i=1}^{n} \sigma_{i}\right)\right|$.

Proof. Since $V$ is an even diagram, $\left\langle\bigcap_{i=1}^{n} \sigma_{i}^{\prime}\right\rangle$ is conjugate to $\langle\sigma\rangle$ for $\sigma \subset \bigcap_{i=1}^{n} \sigma_{i}$. Hence $\left|B^{\prime} \cap\left(\bigcap_{i=1}^{n} \sigma_{i}^{\prime}\right)\right| \leq\left|B \cap\left(\bigcap_{i=1}^{n} \sigma_{i}\right)\right|$, and it remains to show the reverse inequality. We present the case $n=2$. The general case is completely analogous. Assume $\left\langle\sigma_{1} \cap \sigma_{2}\right\rangle$ is conjugate to $\left\langle\bar{\sigma}_{1}^{\prime}\right\rangle$ for $\bar{\sigma}_{1}^{\prime} \subset \sigma_{1}^{\prime}$, and also conjugate to $\left\langle\bar{\sigma}_{2}^{\prime}\right\rangle$ for $\bar{\sigma}_{2}^{\prime} \subset \sigma_{2}^{\prime}$. As $\left\langle\bar{\sigma}_{1}^{\prime}\right\rangle$ is conjugate to $\left\langle\bar{\sigma}_{2}^{\prime}\right\rangle$, if $v \in \bar{\sigma}_{1}^{\prime}$, then there is an odd edge path from $v$ to some vertex of $\bar{\sigma}_{2}^{\prime}$. But if $v \in B^{\prime}$, it belongs only to edges labeled 2. Hence $\bar{\sigma}_{1}^{\prime} \cap B^{\prime}=\bar{\sigma}_{2}^{\prime} \cap B^{\prime}=\bar{\sigma}_{1}^{\prime} \cap \bar{\sigma}_{2}^{\prime} \cap B^{\prime}$. Also, $\left|\sigma_{1} \cap \sigma_{2} \cap B\right|=\left|\bar{\sigma}_{i}^{\prime} \cap B^{\prime}\right|=\left|\bar{\sigma}_{1}^{\prime} \cap \bar{\sigma}_{2}^{\prime} \cap B^{\prime}\right| \leq$ $\left|\sigma_{1}^{\prime} \cap \sigma_{2}^{\prime} \cap B^{\prime}\right|$.

The sets $\sigma_{b^{\prime}}^{\prime} \cap B^{\prime}$ for $b^{\prime} \in \delta^{\prime} \cap B^{\prime}$ cover $B^{\prime}$ (although it is not clear if the sets $\sigma_{b^{\prime}}$ cover $B$ ). Claim 3.2 and the Inclusion-Exclusion Principle imply $\left|B^{\prime}\right| \leq|B|$, and the proof of Proposition 3 is complete.

\section{Circuits OF SIZE 4}

Our goal for this section is to prove the base case of Proposition 4 the case of circuits of length 4 .

Proposition 20. Suppose $(W, S)$ is an even Coxeter system with diagram $V$, and $V^{\prime}$ is another diagram for $W$. If $[x y],[y z],[z v]$ and $[v x]$ are distinct edges of $V^{\prime}$, and $[x y]$ and $[y z]$ have odd labels, then there is a chord (labeled 2) between $v$ and $y$.

Proof. The edges $[v x]$ and $[v z]$ have label 2, by Proposition 2 and Lemma 8 (collapse the edge $[x y]$ to see that $[v z]$ has label 2). By Proposition 2 there is no edge $[x z]$. Suppose $V^{\prime}$ is a minimal counterexample (with respect to $T\left(V^{\prime}\right)$ ). We prove a collection of claims.

\section{Claim 20.1}

(1) Every even label of an edge of $V^{\prime}$ is 2.

(2) If $u \neq y$ is adjacent to $x$ (resp. $z$ ), then $[u x]$ (resp. [uz]) has label 2.

(3) Every odd labeled edge of $V^{\prime}$ is adjacent to $y$ or $v$.

(4) If [yu] (resp. [vu]) has odd label, then $(u v)^{2}=1$ (resp. $\left.(u y)^{2}=1\right)$.

Proof. Otherwise a quotient map leads to a smaller counterexample.

Let $\bar{V}$ be the full subcomplex of $V^{\prime}$ with vertex set $\{v\}$ union the set of all vertices of the odd labeled edges. (In particular, $\{x, y, z, v\} \subset \bar{V}$.)

Claim 20.2 Suppose $\left\langle\sigma^{\prime}\right\rangle$ is conjugate to $\langle\sigma\rangle$ for $\sigma^{\prime}$ and $\sigma$ non-trivial simplices of $V^{\prime}$ and $V$ respectively.

(1) The simplex $\sigma^{\prime}$ contains a vertex of $\bar{V}$.

(2) If $\sigma^{\prime}$ contains a vertex of $V^{\prime}-\bar{V}$, then $\sigma^{\prime}$ contains two vertices of $\bar{V}$.

Proof. If $\sigma^{\prime}$ contains no vertex of $\bar{V}$, then $W / N\left(\sigma^{\prime}\right)$ is a smaller counterexample. If $\sigma^{\prime}$ contains a vertex of $V^{\prime}-\bar{V}$ and $t$ is the only vertex of $\sigma^{\prime} \cap \bar{V}$, then by Lemma $18 W / N\left(\sigma^{\prime}-\{t\}\right)$ is a smaller counterexample.

Claim 20.3 Suppose $\left[x^{\prime} y\right]$ is an odd labeled edge of $V^{\prime}$ (so $\left(x^{\prime} v\right)$ has order 2). Then there is a vertex $t \in \bar{V}$ such that (tv) has odd order, and for every simplex $\sigma^{\prime}$ of $V^{\prime}$ containing $\left\{x^{\prime}, v\right\}$ and such that $\left\langle\sigma^{\prime}\right\rangle$ is conjugate to $\langle\sigma\rangle$ for $\sigma$ a simplex of $V$, $t \in \sigma^{\prime}$. (By Proposition 2, $\sigma^{\prime}$ cannot contain a vertex $t^{\prime} \neq t$ such that $\left(t^{\prime} v\right)$ has odd order. In this sense, $t$ is unique.) 


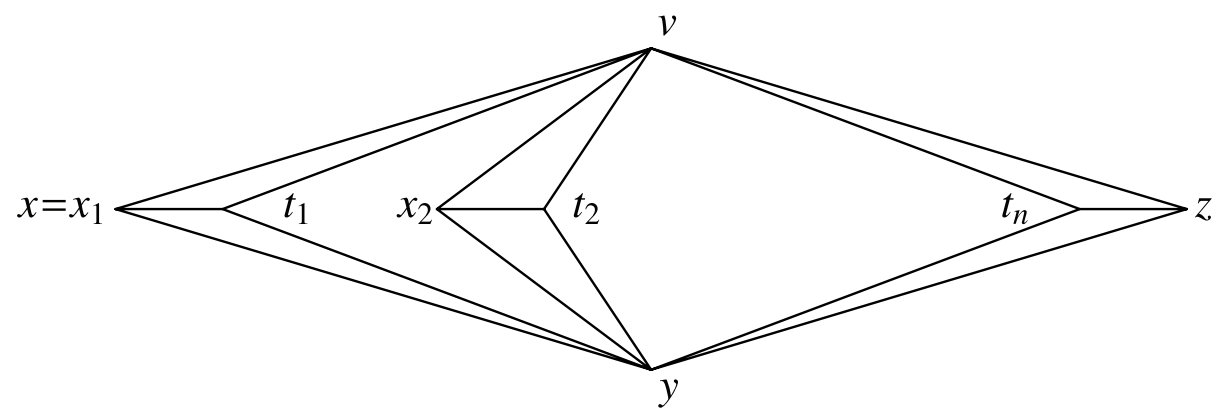

FiguRE 4.

Proof. Suppose $\sigma^{\prime}$ is a simplex of $V^{\prime}$ containing $\left\{x^{\prime}, v\right\}$ and such that $\left\langle\sigma^{\prime}\right\rangle$ is conjugate to $\langle\sigma\rangle$ for some simplex $\sigma$ of $V$; then $\{x, y, z\} \cap \in \sigma^{\prime}=\emptyset$. There is no vertex $t \in \sigma^{\prime}$ such that $[y t]$ is an odd labeled edge by Proposition 2. If there is no vertex $t \in \sigma^{\prime}$ such that $(t v)$ has odd order, then (by Lemma 18) $W / N\left(\left\{x^{\prime} v\right\} \cup\left(\sigma^{\prime}-\left\{x^{\prime}, v\right\}\right)\right)$ is an even Coxeter group and the diagram for this Coxeter group obtained from $V^{\prime}$ by identifying $x^{\prime}$ and $v$, and removing the vertices of $\sigma^{\prime}-\left\{x^{\prime}, v\right\}$, contains a triangle that violates Proposition 2

Now suppose that $\sigma_{1}^{\prime}$ and $\sigma_{2}^{\prime}$ are simplices of $V^{\prime}$ as above, and $t_{i}$ is a vertex of $\sigma_{i}^{\prime}$ such that $t_{i} v$ has odd order and $t_{1} \neq t_{2}$. Then $\left\{x^{\prime}, v\right\} \subset \sigma_{1}^{\prime} \cap \sigma_{2}^{\prime} \equiv \sigma^{\prime}$. But then there is a $t_{3} \in \sigma^{\prime}$ such that $\left(t_{3} v\right)$ has odd order. As $t_{3}$ is in both $\sigma_{1}^{\prime}$ and $\sigma_{2}^{\prime}$, we have a contradiction to Proposition 2 .

Note that in the previous lemma ty has order 2.

By a completely analogous argument we have:

Claim 20.4 Suppose $\left[v t^{\prime}\right]$ is an odd labeled edge of $V^{\prime}$ (so $\left(t^{\prime} y\right)$ has order 2). Then there is a vertex $x^{\prime} \in \bar{V}$ such that $\left(x^{\prime} y\right)$ has odd order, and for every simplex $\sigma^{\prime}$ of $V^{\prime}$ containing $\left\{t^{\prime}, y\right\}$ and such that $\left\langle\sigma^{\prime}\right\rangle$ is conjugate to $\langle\sigma\rangle$ for $\sigma$ a simplex of $V$, $x^{\prime} \in \sigma^{\prime}$. (By Proposition 2, $\sigma^{\prime}$ cannot contain a vertex $x^{\prime \prime} \neq x^{\prime}$ such that $\left(x^{\prime \prime} y\right)$ has odd order. In this sense, $x^{\prime}$ is unique.)

Let $x \equiv x_{1}, x_{2}, \ldots, x_{n}=z$ be the vertices of $\bar{V}$ such that $\left(x_{i} y\right)$ has odd order. Let $t_{i}$ be the vertex of Claim 20.3 for $\left\{x_{i}, v\right\}$, so that $\left(t_{i} v\right)$ has odd order and $\left(t_{i} x_{i}\right)$ has order 2 .

Next we show that $t_{i} \neq t_{j}$ for $i \neq j$. Otherwise, consider simplicies $\sigma_{1}^{\prime}$ and $\sigma_{2}^{\prime}$ containing triangles $\left[t_{i} x_{i} y\right]$ and $\left[t_{i} x_{j} y\right]$ respectively, such that $\left\langle\sigma_{i}^{\prime}\right\rangle$ is conjugate to $\left\langle\sigma_{i}\right\rangle$ for some simplex $\sigma_{i}$ of $V$. But then Claim 20.4 implies $x_{i}=x_{j}$, which is nonsense.

Similarly there is no edge between $t_{i}$ and $x_{j}$ for $i \neq j$ (see Figure 4). Hence we have:

Claim 20.5 The only edges of $\bar{V}$ are $\left[v x_{i}\right],\left[v t_{i}\right],\left[y x_{i}\right],\left[y t_{i}\right]$ and $\left[x_{i} t_{i}\right]$.

Claim 20.6 If $s$ is a vertex of $V^{\prime}-\bar{V}$, then there is no pair of edges $e_{1}, e_{2}$ such that $e_{1}$ connects $s$ to a point of $\left\{x_{i}, t_{i}\right\}$ and $e_{2}$ connects $s$ to a point of $\left\{x_{j}, t_{j}\right\}$ for $i \neq j$. 
Proof. Otherwise let $\sigma_{1}^{\prime}$ and $\sigma_{2}^{\prime}$ be maximal simplices containing $e_{1}$ and $e_{2}$ respectively. Now, $s \in \sigma_{1}^{\prime} \cap \sigma_{2}^{\prime} \equiv \sigma^{\prime}$. By Claim 20.2, $\sigma^{\prime}$ contains two vertices of $\bar{V}$ and so $t_{l}$ or $x_{l}$ is an element of $\sigma^{\prime}$ for some $l$. But this is impossible by Claim 20.5.

Claim 20.7 The set $\{v, y\}$ separates $x$ from $z$.

Proof. Suppose there is an edge path $\left[x s_{1}\right],\left[s_{1} s_{2}\right], \ldots,\left[s_{m} z\right]$ that does not intersect $\{v, y\}$. By Claim 20.6, there is a smallest integer $1<i \leq m$ such that $s_{i}$ is not connected to $x$ or $t_{1}$ by an edge. Note that $s_{i-1} \notin \bar{V}$ and $s_{i-1}$ is connected to $x$ or $t_{1}$ by an edge. Also, by Claim 20.6 (with $s_{i-1}$ in place of $s$ ) $s_{i} \notin \bar{V}$. Let $\sigma^{\prime}$ be a maximal simplex of $V^{\prime}$ containing $\left\{s_{i-1}, s_{i}\right\}$. By Claim 20.2 there is $j \neq 1$ and vertex $u \in\left\{x_{j}, t_{j}\right\} \cap \sigma^{\prime}$. But this is impossible by Claim 20.6 applied to $s_{i-1}$.

By Proposition 11 and Lemma 15 the odd labeled edges of $V^{\prime}$ containing $v$ correspond to edges of $V$ with common vertex $a$ and the odd labeled edges of $V^{\prime}$ containing $y$ correspond to edges of $V$ with common vertex $b$. The subcomplex of $V$ composed of the edges with label $>2$ and adjacent to $a$, and the subcomplex of $V^{\prime}$ composed of the edges with label $>2$ and adjacent to $b$ have trivial intersection by Proposition 12 .

Let $\sigma_{1}^{\prime}$ be a maximal simplex of $V^{\prime}$ containing the triangle $\left[x, t_{1}, v\right]$ and let $\sigma_{2}^{\prime}$ be a maximal simplex of $V^{\prime}$ containing the triangle $\left[x, t_{1}, y\right]$. Let $\sigma_{1}$ and $\sigma_{2}$ be simplices of $V$ such that $\left\langle\sigma_{i}^{\prime}\right\rangle$ is conjugate to $\left\langle\sigma_{i}\right\rangle$. Now, $\sigma_{1}$ contains an edge [ac] (with label $>2$ ) corresponding to $\left[t_{1} v\right]$ and no vertex $c^{\prime} \neq c$ such that $\left[a c^{\prime}\right]$ has label $>2$. Similarly, $\sigma_{2}$ contains an edge $[b d]$ corresponding to $[x y]$ and no vertex $d^{\prime} \neq d$ such that $\left[b d^{\prime}\right]$ has label $>2$. By conjugation we assume that $\left\langle\sigma_{1}\right\rangle=\left\langle\sigma_{1}^{\prime}\right\rangle$ and say $\left\langle\sigma_{2}^{\prime}\right\rangle=w\left\langle\sigma_{2}\right\rangle w^{-1}$ for some $w \in W$. We have $\left\{x, t_{1}\right\} \subset\left\langle\sigma^{\prime}\right\rangle \equiv\left\langle\sigma_{1}^{\prime}\right\rangle \cap\left\langle\sigma_{2}^{\prime}\right\rangle=\left\langle\sigma_{1}\right\rangle \cap w\left\langle\sigma_{2}\right\rangle w^{-1}=g\langle\sigma\rangle g^{-1}$ for $\sigma \subset \sigma_{1}$ and $g \in\left\langle\sigma_{1}\right\rangle$. Since $V$ is even, $\sigma \subset \sigma_{2}$. If $s \in \sigma$ is a vertex of an edge of $V$ with label $>2$, then $s \in\{a, b, c, d\}$. Since $\sigma^{\prime}$ (and hence $\sigma$ ) is right angled, $a$ or $c$ is not in $\sigma$, and $b$ or $d$ is not in $\sigma$. Since $W / N\left(\sigma^{\prime}\right)=W / N(\sigma)$ is right angled, $a$ and $b$ are elements of $\sigma$. We now have that $\langle a, b\rangle$ is isomorphic to $\mathbb{Z}_{2} \times \mathbb{Z}_{2}$.

In a completely analogous manner we find a simplex $\tau^{\prime}$ of $V^{\prime}$ containing $\left\{z, t_{n}\right\}$ such that $\left\langle\tau^{\prime}\right\rangle$ is conjugate to $\langle\tau\rangle$ where $\tau$ is a simplex of $V$ containing $\{a, b\}$. Let $C$ be the component of $V^{\prime}-\{v, y\}$ containing $\left\{x, t_{1}\right\}$ and $D=\Lambda^{\prime}-C$ (so $\left.\left\{z, t_{n}\right\} \subset D\right)$. $W$ decomposes as the amalgamated product $\langle C \cup\{v, y\}\rangle *\langle v, y\rangle\langle D\rangle$. Since $\left\langle x, t_{1}\right\rangle \sim$ $\mathbb{Z}_{2} \times \mathbb{Z}_{2}$ is not a subgroup of a conjugate of the edge group $\langle v, y\rangle \sim \mathbb{Z}_{2} * \mathbb{Z}_{2},\left\langle x, t_{1}\right\rangle$ cannot stabilize a vertex of $\mathcal{T}$ (the Bass-Serre tree for $\langle C \cup\{v, y\}\rangle *\langle v, y\rangle\langle D\rangle$ ), other than $\langle C \cup\{v, y\}\rangle$. Since $\left\{x, t_{1}\right\} \subset g\langle\sigma\rangle g^{-1}$ and since $g\langle\sigma\rangle g^{-1}$ stabilizes some vertex of $\mathcal{T}, g\langle\sigma\rangle g^{-1}$ stabilizes $\langle C \cup\{v, y\}\rangle$. Equivalently, $g\langle\sigma\rangle g^{-1}<\langle C \cup\{v, y\}\rangle$. So $\langle\sigma\rangle<g^{-1}\langle C \cup\{v, y\}\rangle g$ and $\langle\sigma\rangle$ stabilizes the vertex $g^{-1}\langle C \cup\{v, y\}\rangle$ of $\mathcal{T}$. Similarly, $\left\langle z, t_{n}\right\rangle$ only stabilizes the vertex $\langle D\rangle$ of $\mathcal{T}$. As some conjugate $w\langle\tau\rangle w^{-1}$ contains $\left\langle z, t_{n}\right\rangle, w\langle\tau\rangle w^{-1}$ stabilizes $\langle D\rangle$ and so $\langle\tau\rangle$ stabilizes the vertex $w^{-1}\langle D\rangle$ of $\mathcal{T}$. But then $\langle a, b\rangle \sim \mathbb{Z}_{2} \times \mathbb{Z}_{2}$ stabilizes distinct vertices of $\mathcal{T}$. This is impossible as $\mathbb{Z}_{2} \times \mathbb{Z}_{2}$ is not a subgroup of $\mathbb{Z}_{2} * \mathbb{Z}_{2}$. This completes the proof of Proposition 20

Proposition 21. Suppose $W$ is a finitely generated Coxeter group with even diagram $V$ and diagram $V^{\prime}$ which is not even. If in $V^{\prime},[x y]$ has an odd label, $[x u]$ and $[y z]$ have even labels, and $[u z]$ has an odd label, then the circuit (xyzu) has [xz] or $[y u]$ as a chord.

Proof. Otherwise assume $V^{\prime}$ is a minimal counterexample to the proposition. Then we may assume that every even edge of $V^{\prime}$ is labeled 2 and by Proposition 20 
and Lemma 8 there are no odd labeled edges, other than $[x y]$ and $[u z]$. Let $\sigma^{\prime}$ be a maximal simplex of $V^{\prime}$ containing $\{u, x\}$. Then $z, y \notin \sigma^{\prime}$. By Lemma 18, $W / N\left(\{u x\} \cup\left(\sigma^{\prime}-\{u, x\}\right)\right)$ is an even Coxeter group with a diagram obtained from $V^{\prime}$ by identifying $u$ and $x$, and removing the vertices of $\sigma^{\prime}-\{u, x\}$. This contradicts Proposition 2 and the proof is complete.

Lemma 22. Suppose $\mathbb{Z}_{2}^{n}=\left\langle a, b, s_{3}, \ldots, s_{n}\right\rangle=\left\langle a, b, e_{3}, \ldots, e_{n}\right\rangle \equiv G$. Then there is a retraction $h: G \rightarrow\langle a, b\rangle$ such that $h(a)=a, h(b)=b, h\left(e_{i}\right) \in\{a, b\}$, and $h\left(s_{i}\right) \in\{a, 1\}$ for all $i$.

Proof. Let $M_{n, n}$ be the coefficient matrix for $s_{i}$. I.e. $s_{i}=m_{i, 1} a+m_{i, 2} b+m_{i, 3} e_{3}+$ $\cdots+m_{i, n} e_{n}$, where each $m_{i, j} \in\{0,1\}$.

The matrix $M$ is invertible. As the block $\left[m_{i, j}\right]$ for $j>2$ and $i>2$ is invertible, a sequence of elementary row operations (only involving rows 3 through $n$ ) can be used to transform $M$ to the matrix $\bar{M}$ where $\bar{m}_{i, i}=1$ for all $i, \bar{m}_{i, j}=0$ for $i<j$ and for $i>j>2$.

We now define $h$. If $\bar{m}_{i, 1}=1$ and $\bar{m}_{i, 2}=1$, then $h\left(e_{i}\right)=b$.

If $\bar{m}_{i, 1}=1$ and $\bar{m}_{i, 2}=0$, then $h\left(e_{i}\right)=a$.

If $\bar{m}_{i, 1}=0$ and $\bar{m}_{i, 2}=1$, then $h\left(e_{i}\right)=b$.

If $\bar{m}_{i, 1}=0$ and $\bar{m}_{i, 2}=0$, then $h\left(e_{i}\right)=a$.

To understand the effect this has on $h\left(s_{i}\right)$, some notation is helpful. In $\bar{M}$, if $\bar{m}_{i, 1}=1$, then replace it by $1_{a}$. If $\bar{m}_{i, 2}=1$, then replace it by $1_{b}$. If $h\left(e_{i}\right)=a$ (respectively $b$ ) replace $\bar{m}_{i, i}$ by $1_{a}$ (respectively $1_{b}$ ). In each row of $\bar{M}$ (except the second) there are an even number of $1_{b}$-entries. Reversing the above row operations to obtain $M$ from $\bar{M}$ we see that if $\bar{m}_{j, j}=1_{a}$ (respectively $1_{b}$ ), then $m_{i, j} \in\left\{0,1_{a}\right\}$ (respectively $m_{i, j} \in\left\{0,1_{b}\right\}$ ) and $h\left(e_{j}\right)=a$ (respectively $h\left(e_{j}\right)=b$ ). Furthermore, each row of $M$ (except the second) contains an even number of $1_{b}$-entries. I.e. $h\left(s_{i}\right) \in\{1, a\}$.

Proposition 23. Suppose $W$ is a finitely generated Coxeter group with an even diagram $V$ and a diagram $V^{\prime}$ which is not even. If $[x y]$ has odd label in $V^{\prime}$ and (xyst) is a circuit in $V^{\prime}$, then this circuit has $[x s]$ or $[y t]$ as a chord.

Proof. Assume $V^{\prime}$ is a minimal counterexample to the proposition. By Propositions [20 and 21, $[x y]$ is the only odd labeled edge of (xyst). Each even edge of $V^{\prime}$ is labeled 2. By Lemma 8, any odd labeled edge not containing $x$ or $y$ must contain a vertex of $\{s, t\}$, and this edge must be connected to the diagonally opposite vertex of $\{x, y\}$ by an edge labeled 2. By Proposition 20, there can be at most one odd labeled edge containing a given vertex of $\{x, y, s, t\}$.

Case 1. The only odd edge of $V^{\prime}$ is $[x y]$.

By Lemma 18 every simplex $\sigma^{\prime}$ of $V^{\prime}$ such that $\left\langle\sigma^{\prime}\right\rangle$ is conjugate to $\langle\sigma\rangle$ for $\sigma$ a simplex of $V$ is either $\{u\}$ for $u \in\{x, y, s, t\}$ or contains two vertices of $\{x, y, s, t\}$, but not three. By Lemma 19 there is a vertex $u \in V^{\prime}$ such that if $\sigma^{\prime}$ is a simplex of $V^{\prime}$ containing $u$ and $\left\langle\sigma^{\prime}\right\rangle$ is conjugate to $\langle\sigma\rangle$ for $\sigma$ a simplex of $V$, then $\{x, y\} \subset \sigma^{\prime}$, and if $\tau^{\prime}$ is a simplex of $V^{\prime}$ containing $\{x, y\}$ and $\left\langle\tau^{\prime}\right\rangle$ is conjugate to $\langle\tau\rangle$ for $\tau$ a simplex of $V$, then $\tau^{\prime}$ contains $u$.

We show that $\{x, y\}$ separates $u$ from $t$. Otherwise, choose a shortest edge path avoiding $\{x, y\}$ from $u$ to $t$. Let $v$ be the first vertex of this path that is not adjacent to both $x$ and $y$ and let $v^{\prime}$ be the previous vertex. Choose a maximal simplex $\sigma_{1}^{\prime}$ containing $\left\{x, y, v^{\prime}\right\}$ and a maximal simplex $\sigma_{2}^{\prime}$ containing $\left\{v, v^{\prime}\right\}$. Let 
$\sigma^{\prime}=\sigma_{1}^{\prime} \cap \sigma_{2}^{\prime}$. Assume $\sigma_{2}^{\prime}$ does not contain $x$. Then $\sigma^{\prime}$ does not contain $x, s$ or $t$, which is impossible.

Now as $\{x, y\}$ separates $V^{\prime}, 7$ implies that $W$ visually decomposes (with respect to $\left.V^{\prime}\right)$ as $\langle A\rangle *\langle x, y\rangle\langle B\rangle$, where $A \cup B$ is the vertex set of $V^{\prime}$ and $A$ and $B$ properly contain $\{x, y\}$. By [7], there is a non-trivial visual (with respect to $V$ ) decomposition $C *_{E} D$ of $W$ such that $E$ is a subgroup of a conjugate of $\langle x, y\rangle$. As $V$ is even, $E$ is a proper subgroup of a conjugate of $\langle x, y\rangle$. Then $W$ visually decomposes non-trivially (with respect to $V^{\prime}$ ) as $\langle F\rangle *\langle H\rangle\langle G\rangle$ where $\langle H\rangle$ is a subgroup of a conjugate of $E$ and hence $\langle H\rangle$ is conjugate to a proper subgroup of $\langle x, y\rangle$. This implies $H$ is trivial $\{x\}$ or $\{y\}$. We have that $F \cup G$ is the vertex set of $V^{\prime}$ and $H$ is a proper subset of $F$ and $G$. As $\langle x, y, s, t\rangle$ is 1 -ended, we must have $\{x, y, s, t\}$ as a subset of $F$ or $G$. Assume $\{x, y, s, t\} \subset F$. Let $e$ be a vertex of $G-H$ and let $\sigma^{\prime}$ be a maximal simplex of $V^{\prime}$ containing $e$. Then $\sigma^{\prime} \cap\{x, y, s, t\}$ is either $\emptyset$ or $v^{\prime}$. This is impossible as $\sigma^{\prime}$ must contain two vertices of $\{x, y, s, t\}$. Case 1 is complete.

Hence there must be an odd edge at $s$ or $t$.

Case 2. Assume there is an odd edge $[t v]$ but no odd edge at $s$.

There is an edge $[y v]$ labeled 2 by Lemma 8 and an edge $[v x]$ labeled 2 by Proposition [21. There are no odd labeled edges, other than $[x y]$ and $[v t]$. Every simplex $\sigma^{\prime}$ (with at least two vertices) such that $\left\langle\sigma^{\prime}\right\rangle$ is conjugate to $\langle\sigma\rangle$ for some simplex $\sigma$ of $V$ must contain at least two vertices of $\{x, y, s, t, v\}$. If there is an edge $[s v]$, then it is labeled 2. By Lemma 19 there is a vertex $u$ of $V^{\prime}$ such that if $\sigma^{\prime}$ is a simplex of $V^{\prime},\left\langle\sigma^{\prime}\right\rangle$ is conjugate to $\langle\sigma\rangle$ for some simplex $\sigma$ of $V$ and $\{x, y\} \subset \sigma^{\prime}$, then $u \in \sigma^{\prime}$, and if $\alpha^{\prime}$ is a simplex of $V^{\prime},\left\langle\alpha^{\prime}\right\rangle$ is conjugate to $\langle\alpha\rangle$ for some simplex $\alpha$ of $V$ and $u \in \alpha^{\prime}$, then $\{x, y\} \subset \alpha^{\prime}$. Note that $u \neq v$.

Let $[a b]$ and $[c d]$ be the edges of $V$ corresponding to (see Theorem [7) [xy] and $[t v]$ respectively. Note that $\{a, b\} \cap\{c, d\}=\emptyset$ by Proposition 12 .

Claim 23.1 $\{x, y, v\}$ separates $u$ from $t$ (and $s$ ).

Proof. Otherwise, there are consecutive vertices $u=u_{0}, \ldots, u_{n}=t$ such that no $u_{i} \in\{x, y, v\}$. Assume that $i$ is the first integer such that $u_{i}$ does not commute with $x$ and $y$. Suppose $u_{i}$ does not commute with $x$ (the case that $u_{i}$ does not commute with $y$ is completely analogous). Let $\sigma^{\prime}=\sigma_{1}^{\prime} \cap \sigma_{2}^{\prime}$ where $\sigma_{1}^{\prime}$ is a maximal simplex containing $\left\{x, y, u_{i-1}\right\}$ and $\sigma_{2}^{\prime}$ is a maximal simplex containing $\left\{u_{i-1}, u_{i}\right\}$. Then $\{t, s, x\} \cap \sigma^{\prime}=\emptyset$ and so $\left\{u_{i-1}, y, v\right\} \subset \sigma^{\prime}$.

Observe that $\sigma^{\prime}-\{y\} \subset l k_{2}(x, y)$.

Suppose $\sigma$ is a simplex in $V$ such that $\langle\sigma\rangle$ is conjugate to $\left\langle\sigma^{\prime}\right\rangle$. By conjugation, we may assume that $\langle\sigma\rangle=\left\langle\sigma^{\prime}\right\rangle$. We may assume that $a, c \in \sigma$ and $b, d \notin \sigma$, since $\langle\sigma\rangle$ is abelian. Let $N=N(\sigma-\{a, c\})$. Note that $y, v \notin N$.

Now we show:

$$
\text { If } p \in\left\langle\sigma^{\prime}-\{y\}\right\rangle \text {, then } y p \notin N \text {. }
$$

Suppose $y p \in N$. As yp conjugates $x y\left(=w(a b)^{2} w^{-1}\right)$ to $y x, w^{-1} y p w$ conjugates $(a b)^{2}$ to $(b a)^{2}$. Proposition 10 implies that $w^{-1} y p w=e f$ for $e \in l k_{2}(a, b)$ and $f$ of odd length in $\langle a, b\rangle$. As yp $\in\langle\sigma\rangle$, yp $=a g$ for $g \in\langle\sigma-\{a\}\rangle$. (Otherwise, yp $=g \in\langle\sigma-\{a\}\rangle$ implying ef $=w^{-1} g w$ implying (the odd length element of $\langle a, b\rangle) f=e w^{-1} g w$. But $e w^{-1} g w \in N(S-\{a, b\})$ so this is impossible.) But $a g \notin N$ and $(*)$ is proved.

Let $q: W \rightarrow W / N$ be the quotient map. Note that $q\left\langle\sigma^{\prime}\right\rangle=q\langle\sigma\rangle=\langle q(a), q(c)\rangle \equiv$ $\mathbb{Z}_{2} \times \mathbb{Z}_{2}$. As $y, v$ and $y v$ are not elements of $N, q\left\langle\sigma^{\prime}\right\rangle=\langle q(y), q(v)\rangle$. To finish the 
claim, we show that $W / N$ is a smaller counterexample. (Note that $N$ is non-trivial, as $u_{i-1} \in \sigma^{\prime}-\{y, v\}$.)

First we show that if $m \in \sigma^{\prime}-\{y, v\}$, then $q(m) \in\{1, q(v)\}:$ If $q(m) \neq 1$, then $q(m) \in\{q(y), q(v), q(y v)\}$. If $q(m)=q(y)$, then $q(m y)=1$ implying $m y \in N$, contrary to $(*)$. If $q(m)=q(y v)$, then $q(m v y)=1$ implying $m v y \in N$, contrary to $(*)$. Hence $q(m)=q(v)$.

If $K$ is the kernel of the restriction of $q$ to $\left\langle\sigma^{\prime}\right\rangle$, then the normal closure of $K$ in $W$ is $N$. As $K$ is generated by $\left\{m \in \sigma^{\prime}: q(m)=1\right\} \cup\left\{m v: m \in \sigma^{\prime}\right.$ and $q(m)=q(v)\}$, a diagram for the even Coxeter group $W / N$ is obtained from $V^{\prime}$ by removing the vertices of $\left(\sigma^{\prime}-\{y, v\}\right) \cap \operatorname{ker}(q)$ and identifying the remaining vertices of $\sigma^{\prime}-\{y, v\}$ with $v$.

We can now finish Case 2. By [7] there is a simplex $\sigma$ in $V$ such that $\sigma$ separates $V$ and $\langle\sigma\rangle$ is a subgroup of a conjugate of $\langle x, y, v\rangle$. Note that the edge $[a b]$ corresponding to $[x y]$ is not in $\sigma$. (Otherwise, the pigeon-hole principle implies $\sigma=\{a, b\}$ and $\langle a, b\rangle$ is conjugate to $\langle x, y, v\rangle$. This is impossible since $u \notin \sigma^{\prime}$.) The edge $[c d]$ corresponding to $[t v]$ is not in $\sigma$. (Otherwise the image of $\langle c, d\rangle$ in $W / N(x y)\left(=W / N\left((a b)^{2}\right)\right)$ is abelian. But $\langle c, d\rangle$ injects under this quotient.) Hence $\langle\sigma\rangle$ is abelian. For $k$ odd, the order 2-elements of the dihedral group $D_{k}$ are precisely the odd length elements. It is straightforward to see (for odd $k$ ) that $D_{k}$ does not contain a copy of $\mathbb{Z}_{2} \times \mathbb{Z}_{2}$. Hence $\langle\sigma\rangle$ is either isomorphic to $\mathbb{Z}_{2}$ or $\mathbb{Z}_{2} \times \mathbb{Z}_{2}$. Again applying [7, either an edge (labeled 2) or a vertex of $V^{\prime}$ must separate $V^{\prime}$ and the group generated by the vertices of this separating set is a subgroup of a conjugate of $\langle x, y, v\rangle$.

We next show no vertex $w$ of $V^{\prime}$ can separate $V^{\prime}$. Otherwise, there is a component $C$ of $V^{\prime}-\{w\}$ such that $\{x, y, s, t, v\} \subset C \cup\{w\}$ (the group $\langle x, y, s, t, v\rangle$ is 1-ended). But if $k$ is a vertex of a component $K \neq C$ of $V^{\prime}-\{w\}$, then a maximal simplex of $V^{\prime}$ containing $k$ would contain at most one vertex of $\{x, y, s, t, v\}$, which is impossible.

If an edge labeled 2 separates $V^{\prime}$ and the group for this edge is conjugate to a subgroup of $\langle x, y, v\rangle$, then the only candidates are the edges $[x v],[y v]$ and $[x t]$. The groups for these edges are all conjugate. All cases are completely similar, and we assume $[y v]$ separates $V^{\prime}$. Then (by [7]) there is an edge separating $V$ such that the corresponding group is conjugate to $\langle y, v\rangle$. We may assume that this edge is $[a c]$. By conjugation, we may assume that $\langle y, v\rangle=\langle a, c\rangle$. Note that $v y$ conjugates $x y\left(=w_{1}(a b)^{2} w_{1}^{-1}\right)$ to $y x\left(=w_{1}(b a)^{2} w_{1}^{-1}\right)$.

If $v y=c$, then $c w_{1}(a b)^{2} w_{1}^{-1} c=w_{1}(b a)^{2} w_{1}^{-1}$. But in $W / N(\{c\}),(a b)^{2} \neq(b a)^{2}$, so this is impossible. If $v y=a$, then choose $g \in\langle x, y\rangle$ such that $g v y g^{-1}=x v$. As $x v$ conjugates $t v\left(=w_{2}(c d)^{2} w_{2}^{-1}\right)$ to $v t\left(=w_{2}(d c)^{2} w_{2}^{-1}\right), g a g^{-1}$ conjugates $w_{2}(c d)^{2} w_{2}^{-1}$ to $w_{2}(d c)^{2} w_{2}^{-1}$. But in $W / N\left(\{a\},(c d)^{2} \neq(d c)^{2}\right.$, so this is impossible. The only other possibility is $v y=a c$. Hence we have $\{y, v\}=\{a, c\}$.

The group $\langle x, y, s, t, v\rangle$ is 1-ended and hence $\{x, y, s, t, v\}$ is a subset of $\{v, y\}$ union a component $C$ of $V^{\prime}-\{v, y\}$. Let $\sigma^{\prime}$ be a maximal simplex containing a vertex of a component of $V^{\prime}-\{v, y\}$ other than $C$. Then $\sigma^{\prime}$ must also contain $\{v, y\}(=\{a, c\})$ and each edge label of $\sigma^{\prime}$ is 2. Assume that $\left\langle\sigma^{\prime}\right\rangle=w\langle\sigma\rangle w^{-1}$ for $\sigma$ a simplex of $V$. Then $a, c \in \sigma, w a w^{-1}=a$ and $w c w^{-1}=c$. We wish to apply Lemma 22 to obtain a smaller counterexample.

Write $\left\langle\sigma^{\prime}\right\rangle=\left\langle a, c, e_{3}, \ldots, e_{n}\right\rangle=\left\langle a, c, w s_{3} w^{-1}, \ldots, w s_{n} w^{-1}\right\rangle=\left\langle w \sigma w^{-1}\right\rangle$ and suppose $h$ is the retraction of this group to $\langle a, c\rangle$ defined in Lemma 22. By the definition of $\sigma^{\prime}, \operatorname{ker}(h)$ is non-trivial. If $h\left(w s_{i} w^{-1}\right)=a$, then observe that 


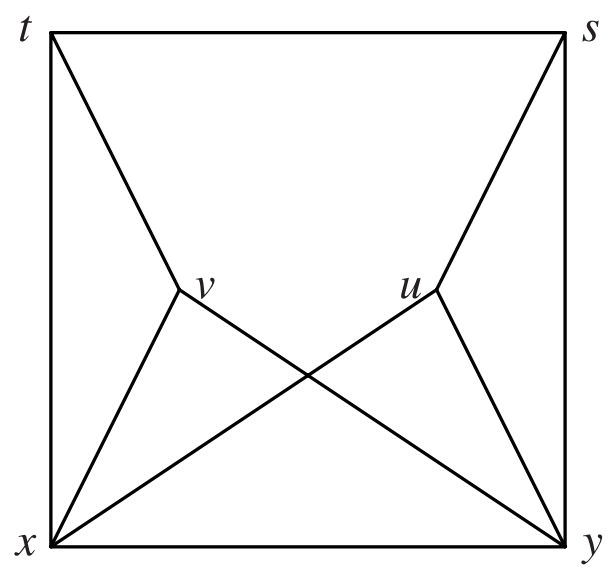

FiguRE 5.

$w s_{i} w^{-1} a=w\left(s_{i} a\right) w^{-1}$. Thus, an even diagram for $W / N(k e r(h))$ is obtained from $V$ by removing all $s_{i}$ such that $h\left(w s_{i} w^{-1}\right)=1$ and identifying $s_{i}$ with $a$ if $h\left(w s_{i} w^{-1}\right)=a$. Another diagram for $W / N(\operatorname{ker}(h))$ is obtained from $V^{\prime}$ by identifying $e_{i}$ with $v$ when $h\left(e_{i}\right)=v$ and identifying $e_{j}$ with $y$ when $h\left(e_{j}\right)=y$. Then $W / N(k e r(h))$ is a smaller counterexample, finishing Case 2 .

Now the final case.

Case 3. Assume there are edges $[t v]$ and $[s u]$ with odd labels.

There are edges $[u y][u x][v y]$ and $[v x]$ with labeled 2. (See Figure 5.) Let $\{a, b, c, d, e, f\} \subset V$ be vertices such that the edge correspondence of Theorem 7 relates $[x y]$ to $[a b],[t v]$ to $[c d]$ and $[u s]$ to $[e f]$. By Proposition [12, the edges $[a b],[c d]$ and $[e f]$ are mutually disjoint. By Theorem 17 there is an edge $[g h] \in$ $\{[a b],[c d],[e f]\}$ such that every other edge of $V$ containing $g$ is labeled 2 and such that if $[g k]$ is such an edge, then $[k h]$ is also an edge of $V$. We call $g$ a special vertex of $[g h]$. A quotient argument shows that each of $[a b],[c d],[e f]$ contains a special vertex. E.g. if the edge labels on $[c d]$ and $[e f]$ are changed to 2, then Theorem 17 implies that $[a b]$ must have a special vertex. The next claim implies that there cannot be an edge $[u v]$ in the minimal counterexample.

Lemma 24. Suppose $(W, S)$ is a finitely generated even Coxeter system, and $V^{\prime}$ is a diagram for $W$ with non-intersecting odd edges $[x y]$, [tv] and [us], and even edges $[x u],[x v],[x t][y u],[y s],[y v],[t s]$ and $[u v]$ (and so a tetrahedron $[x y u v]$ and triangles $[x t v]$ and $[$ suy $]$ ). Then there is an edge (labeled 2) $[y t]$ or $[x s]$.

Proof. If an edge not listed in the hypothesis connects two vertices of $\{x, y, u, v, t, s\}$, it must have label 2 by Proposition 2. Assume $V^{\prime}$ is a minimal counterexample to the lemma. Let $V$ be the even diagram for $(W, S)$, and assume the edges $[x y]$, $[t v]$ and $[s u]$ of $V^{\prime}$ correspond to the edges $[a b],[c d]$ and [ef], respectively, of $V$. Again, $[a b],[c d]$ and $[e f]$ are mutually disjoint. All even edges of $V^{\prime}$ are labeled 2 by the minimality of $V^{\prime}$. Every odd edge of $V^{\prime}$ contains a vertex of $\{x, y, u, v, t, s\}$ (otherwise collapse for a smaller counterexample). Suppose $V^{\prime}$ contained an odd edge other than $[x y]$, $[t v]$ or $[u s]$. Then this edge must contain $x, y, s$ or $t$ (otherwise collapse). If $[x w]$ is an odd edge for $w \neq y$, there must be an edge $[w s]$ (or collapsing would give a smaller counterexample). In this situation, Proposition 20 implies 

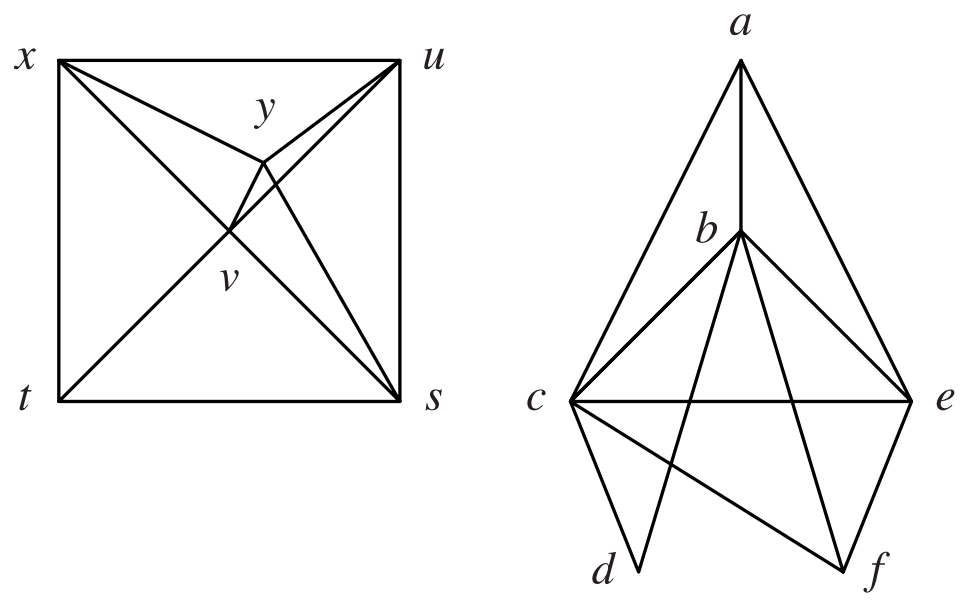

FiguRE 6.

there is an edge $[x s]$ and we are finished. Similarly if there is an odd edge at $y, s$ or $t$. Hence we may assume there is no odd edge of $V^{\prime}$ other than $[x y],[t v]$ and $[u s]$.

We assume $a, d$ and $f$ are special vertices of $[a b],[c d]$ and $[e f]$ respectively. Observe that if $\sigma$ is a simplex of $V$ containing $a$, then $\sigma \cup\{b\}$ is a simplex of $V$. Similarly for $d$ and $f$. Let $\sigma_{1}^{\prime}$ be a maximal simplex of $V^{\prime}$ containing $\{x, y, v, u\}$. Then $\left\langle\sigma_{1}^{\prime}\right\rangle$ is conjugate to $\left\langle\sigma_{1}\right\rangle$ for $\sigma_{1}$ a (maximal) simplex of $V$. Now $\{a, b\} \subset \sigma_{1}$. Either $c$ or $d$ is an element of $\sigma_{1}$, but not both (as $t \notin \sigma_{1}^{\prime}$ ). Hence $c \in \sigma_{1}$. Similarly, $e \in \sigma_{1}$. Note that $\{a, b\}$ does not commute with $\{c, d\}$ or $\{e, f\}$. Considering the triangles $[y u s]$ and $[t v x]$, we see that $\{b\}$ commutes with $\{e, f, c, d\}$.

Consider the circuit (tvus). By Proposition 21, there must be an edge (chord) $[t u]$ or $[v s]$. If both exist, then $\{c, d\}$ commutes with $\{e, f\}$ and there is a maximal simplex of $V$ containing $\{b, c, d, e, f\}$, implying there is a maximal simplex in $V^{\prime}$ containing $\{t, v, u, s\}$ and either $x$ or $y$, which is impossible in our minimal counterexample.

Now suppose $[v s]$ is an edge of $V^{\prime}$, but $[t u]$ is not. (See Figure 6.) Let $\sigma_{2}^{\prime}$ be a maximal simplex of $V^{\prime}$ containing $\{u, s, y, v\}$. Then $\left\langle\sigma_{2}^{\prime}\right\rangle$ is conjugate to $\left\langle\sigma_{2}\right\rangle$ for $\sigma_{2}$ a (maximal) simplex of $V$ and $\{e, f, b, c\} \subset \sigma_{2}$. Choose a maximal simplex $\sigma_{3}^{\prime}$ of $V^{\prime}$ containing $\{t, v, x\}$. Then there exists $\sigma_{3}$, a maximal simplex of $V$ containing $\{c, d, b\}$ and such that $\left\langle\sigma_{3}^{\prime}\right\rangle$ is conjugate to $\left\langle\sigma_{3}\right\rangle$. Let $\sigma_{4}^{\prime}$ be a maximal simplex of $V^{\prime}$ containing $\{t, v, s\}$. Then there exists $\sigma_{4}$, a maximal simplex of $V$ containing $\{c, d\}$ and either $f$ or $e$, and such that $\left\langle\sigma_{4}^{\prime}\right\rangle$ is conjugate to $\left\langle\sigma_{4}\right\rangle$. But then either $\{b, c, d, f\}$ or $\{b, c, d, e\}$ is a simplex. This implies there is a simplex $\sigma^{\prime}$ of $V^{\prime}$ containing $\{t, v\}$, a vertex of $\{x, y\}$ and a vertex of $\{u, s\}$. This is impossible, and $[v s]$ is not an edge of $V^{\prime}$.

Next assume that $[t u]$ is an edge, but $[s v]$ is not an edge of $V^{\prime}$. (See Figure 7.) We may assume that $\{x, y, u, v\} \subset \sigma_{1}^{\prime}$ and $\{a, b, c, e\} \subset \sigma_{1}$ as above. Also, $\{x, u, v, t\} \subset$ $\sigma_{2}^{\prime}$ and $\{c, d, b, e\} \subset \sigma_{2} ;\{u, s, y\} \subset \sigma_{3}^{\prime}$ and $\{e, f, b\} \subset \sigma_{3}$; and $\{t, u, s\} \subset \sigma_{4}^{\prime}$ and $\sigma_{4}$ contains $\{e, f\}$ and either $c$ or $d$. But then either $\{e, f, c, b\}$ or $\{e, f, d, b\}$ is a simplex. 

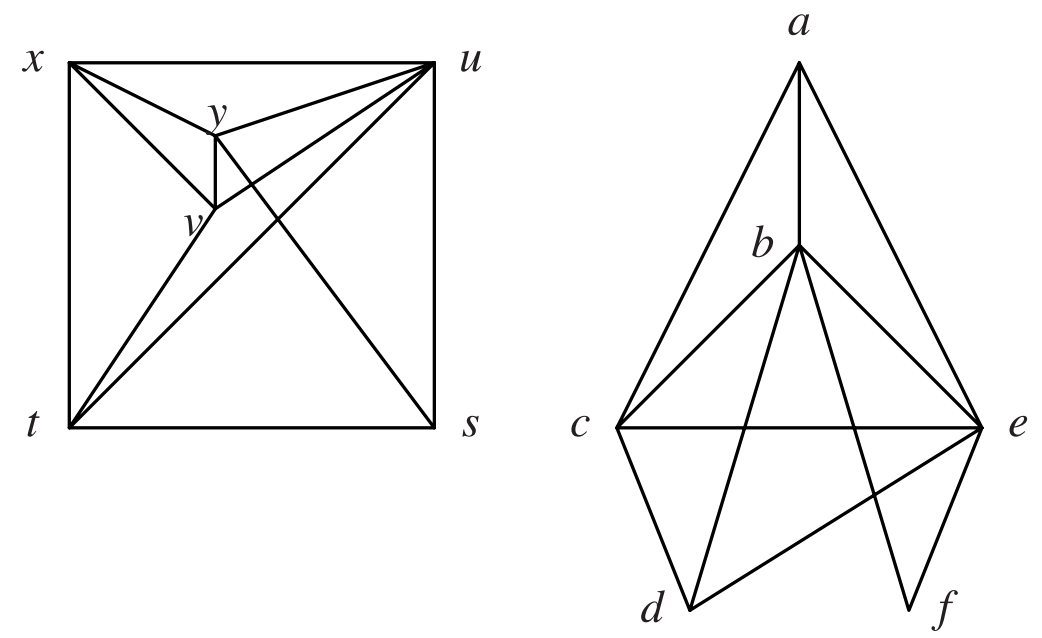

FIGURE 7.

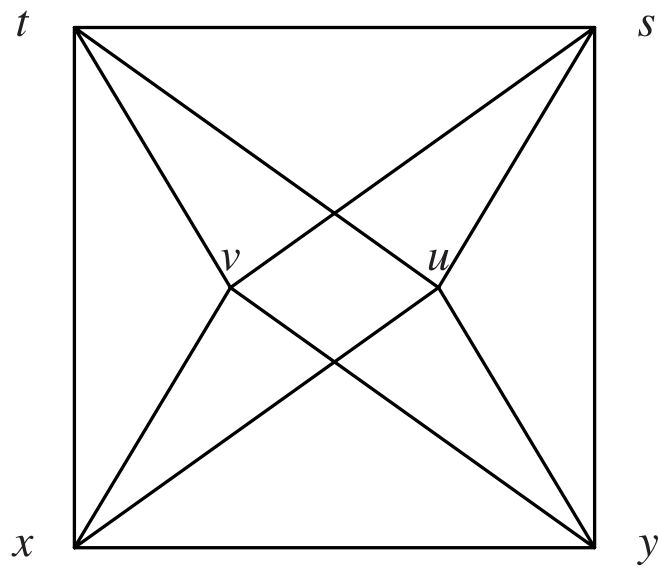

FiguRE 8

This implies there exists a simplex $\sigma^{\prime}$ of $V^{\prime}$ containing $\{u, s\}$, a vertex of $\{x, y\}$ and a vertex of $\{v, t\}$. This is impossible.

Remark 5. Consider the circuit (suxt). There must be an (labeled 2) edge [ut] or $[u v]$, or collapsing $[v t]$ gives a smaller counterexample. Consider the circuit (syvt). There must be an (labeled 2) edge [vs] or $[u v]$, or collapsing [us] gives a smaller counterexample. Either of these observations (along with Lemma 24) could be used in conjunction with the ideas of $\S 7$ to complete a proof of Case 3 .

Lemma 24 implies $[u v]$ is not an edge of $V^{\prime}$. At this point Figure 8 is our model. If $\sigma^{\prime}$ is a simplex of $V^{\prime}$ such that $\left\langle\sigma^{\prime}\right\rangle$ is conjugate to $\langle\sigma\rangle$, for $\sigma$ a simplex of $V$, and $\sigma^{\prime}$ contains a vertex of $V^{\prime}-\{x, y, t, s, u, v\}$, then $\sigma^{\prime}$ must contain two vertices of $\{x, y, t, s, u, v\}$. 
Claim 23.2 The set $\{x, y, u, v\}$ separates $V^{\prime}$.

Proof. Suppose not. By Lemma 19, there is a vertex $z$ of $V^{\prime}-\{x, y\}$ such that every simplex $\sigma^{\prime}$ of $V^{\prime}$ containing $[x y]$ and such that $\left\langle\sigma^{\prime}\right\rangle$ is conjugate to $\langle\sigma\rangle$ for $\sigma$ a simplex of $V$, contains $z$, and every simplex $\sigma^{\prime}$ of $V^{\prime}$ containing $z$ such that $\left\langle\sigma^{\prime}\right\rangle$ is conjugate to $\langle\sigma\rangle$ for $\sigma$ a simplex of $V$, contains [xy]. Clearly $z \notin\{v, u, t, s\}$. Suppose $z=z_{0}, z_{1}, \ldots, z_{n}=t$ are consecutive vertices of $V^{\prime}-\{x, y, u, v\}$. Assume that $i$ is the first integer such that $z_{i}$ is not adjacent to both $x$ and $y$. Both cases are analogous, and we assume that $z_{i}$ is not adjacent to $y$. Let $\sigma_{1}^{\prime}$ be a maximal simplex of $V^{\prime}$ containing $\left\{x, y, z_{i-1}\right\}$, and $\sigma_{2}^{\prime}$ a maximal simplex containing $\left\{z_{i-1}, z_{i}\right\}$. Let $\sigma^{\prime}=\sigma_{1}^{\prime} \cap \sigma_{2}^{\prime}$. Then $\{y, s, t\} \cap \sigma^{\prime}=\emptyset$. Then $\sigma^{\prime}$ must contain $z_{i-1}$ and either $\{x, u\}$ or $\{x, v\}(\{v, u\}$ is not a possibility by Lemma 24). Both cases have analogous proofs and we assume $\{x, u\} \subset \sigma^{\prime}$. Say $\sigma$ is a simplex of $V$ and $\langle\sigma\rangle$ is conjugate to $\left\langle\sigma^{\prime}\right\rangle$. Then we may assume $b, e \in \sigma$ and $a, c, d, f \notin \sigma$. Let $N=N(\sigma-\{b, e\})$. Each vertex of $\sigma^{\prime}-\{x\}$ commutes with $x$ and $y$. An argument completely analogous to that for statement $(*)$ in the proof of Claim 23.1 implies that if $p \in\left\langle\sigma^{\prime}-\{x\}\right\rangle$, then $x p \notin N$. Just as in the argument following the proof of $(*)$, this implies $W / N$ is a smaller counterexample.

By [7] there is a full subgraph $A$ separating $V$ such that $\langle A\rangle$ is conjugate to a subgroup of $\langle u, v, x, y\rangle=\langle u, x, y\rangle *\langle x, y\rangle\langle v, x, y\rangle$. The edge [ab] is not in $A$, for otherwise, a conjugate of $\langle a, b\rangle$ is a subgroup of $\langle u, x, y\rangle$ or $\langle v, x, y\rangle$. But all three of these groups have the same order. This would imply $\langle a, b\rangle$ is conjugate to $\langle u, x, y\rangle$ or $\langle v, x, y\rangle$, but clearly $N(\{a, b\})$ is not equal to $N(\{u, x, y\})$ or $N(\{v, x, y\})$. As in Case 2 (consider $W / N(x y)$ ) neither $[c d]$ nor $[e f]$ is an edge of $A$. So, $A$ is right angled. The group $\langle x, y, u, v\rangle=\langle x, y, v\rangle *\langle x, y\rangle\langle x, y, u\rangle$ is 2-ended and contains no copy of $\mathbb{Z}_{2} \times \mathbb{Z}_{2} \times \mathbb{Z}_{2}$. This implies that $\langle A\rangle=1, \mathbb{Z}_{2}, \mathbb{Z}_{2} \times \mathbb{Z}_{2}, \mathbb{Z}_{2} * \mathbb{Z}_{2}$, or $\mathbb{Z}_{2} \times \mathbb{Z}_{2} * \mathbb{Z}_{2} \mathbb{Z}_{2} \times \mathbb{Z}_{2}$. Using [7] again, a subset $B$ of the vertices of $V^{\prime}$ separates $V^{\prime}$ such that $\langle B\rangle$ is right angled and a subgroup of a conjugate of $\langle A\rangle$ and so a subgroup of a conjugate of $\langle x, y, u, v\rangle$. This implies that $B$ is a subset of $\{u, x, v\},\{u, y, v\}$, $\{x, t\}$, or $\{y, s\}$. By Corollary 6 of [7], we may assume that $\langle A\rangle$ is conjugate to $\langle B\rangle$. Note that $\{x, y, u, v, s, t\}$ is a subset of $B$ union a component of the complement of $B$. Select a maximal simplex $\sigma^{\prime}$ intersecting another component of the complement of $B$. Then $\sigma^{\prime} \cap B$ must be equal to $\{x, t\},\{x, v\},\{x, u\},\{y, v\},\{y, s\}$ or $\{y, u\}$ and be conjugate to a simplex of $V$. Now proceed as in Case 2, to finish Case 3 and Proposition 23,

\section{The Proof of Proposition 4}

In this section we finish the proof of Proposition 4. Throughout this section, we assume that $V^{\prime}$ is a smallest diagram for an even Coxeter group such that $V^{\prime}$ contains a circuit $l$ without chords, the length of $l$ is $\geq 5$ and $l$ contains an odd labeled edge $[x y]$. By the minimality of $V^{\prime}$, all even edges of $V^{\prime}$ are labeled 2. By Lemma 8, Proposition 23 and the minimality of $V^{\prime}$, all edges of $l$ other than $[x y]$ are labeled 2.

Claim 4.1 If $l^{\prime}$ is a circuit of $V^{\prime}$ containing 2 odd labeled edges, then $l^{\prime}$ has a chord.

Proof. Otherwise, the diagram obtained from $V^{\prime}$ by collapsing one of the edges of $l^{\prime}$ contradicts Proposition 23 or is a smaller example than $V^{\prime}$.

Claim 4.2 If $[x u]$ has odd label, then $u=y$. 
Proof. By Lemma 8, $u$ is connected to a vertex of $l-s t(x)$ by an edge. Say the consecutive vertices of $l$ are $x=a_{0}, a_{1}, \ldots, a_{n}=y$. Let $i$ be the largest integer such that $\left[u a_{i}\right]$ is an edge of $V^{\prime}$. By Propositions 2 and 20, $i<n-1$. The circuit with consecutive vertices $\left(y x u a_{i} \ldots a_{n-1}\right)$ has no chord, contradicting Claim 4.1.

Claim 4.3 If $[u v] \neq[x y]$ is an odd labeled edge, then $[u v]$ has one vertex on $l$ and the other vertex in $l k_{2}(x, y)$.

Proof. If neither $u$ nor $v$ is a vertex of $l$, then $W / N(u v)$ is a smaller example. Assume $v$ is a vertex of $l$. If $v$ is not adjacent to $x$ or $y$ (i.e. no edge of $V^{\prime}$ connects $v$ to $x$ or $y)$ and $u \notin l k_{2}(x, y)$, then the quotient of $W$ by $N(u v)$ gives a smaller example. If $v$ is adjacent to $x$, then $u$ is not a vertex of $l$ (as $l$ has no chord), but $u$ is adjacent to $y$ or again $W / N(u v)$ is a smaller example. Now by Claim 4.1 (applied to the circuit (vuyx)), $u$ is adjacent to $x$.

Claim 4.4 If $[u v]$ is an odd labeled edge of $V^{\prime}$, then $[u v]$ is contained in a circuit of length $\geq 5$ without chords.

Proof. Suppose otherwise. We assume that $v \in l$ and $u \in l k_{2}(x, y)$. If $s, t$ are the vertices of $l$ adjacent to $v$, then $s, t \in l k_{2}(u)$ or $[u v]$ belongs to a circuit of length $\geq 5$ without chords (all edges of this path not containing $u$ are in $l$ ). Note that no vertex of $l-\{s, t\}$ belongs to $l k_{2}(v)$ (and so no such vertex belongs to $l k_{2}(u, v)$ ).

Next we show that if $[v w]$ is such that $w \neq u$ and $w \notin l k_{2}(u, v)$ (equivalently, $w$ is not adjacent to $u$ ), then there is no edge path in $V^{\prime}-\left(l k_{2}(u, v) \cup\{v\}\right)$ connecting $w$ to $u$ or a vertex of $l$. Otherwise, there is a circuit containing [uv] and avoiding $l k_{2}(u, v)$. A shortest such circuit has length $\geq 5$ by Propositions 2 and 23, contrary to the original supposition.

Now, let $U$ be the union of all components $K$ of $V^{\prime}-\left(l k_{2}(u, v) \cup\{v\}\right)$ such that $u \notin K$ and for some vertex $w \in K,[w v]$ is an edge. Note that there is no edge connecting a vertex of $U$ to $u$ and $U \cap l=\emptyset$. Twist $U$ around [uv] to form the diagram $V^{\prime \prime}$ for $W$. If $z \neq u$ and $[v z]$ is an edge of $V^{\prime \prime}$, then $z \in l k_{2}(u, v)$. By Proposition 3 there is a vertex $w(\notin\{s, t\})$ of $V^{\prime \prime}$ such that the triangle [uvw] can be replaced by an edge $[u z]$ with label 2 times the label of $[u v]$. The resulting diagram for $W$ is smaller than $V^{\prime}$ and contains a faithful copy of $l$ (with $v$ replaced by $z)$.

Claim 4.5 If $v$ is a vertex of $l$ not adjacent to $x$ or $y$, then there is no odd edge at $v$.

Proof. Assume that $[u v]$ is such an odd edge. By Claim 4.3, $u \in l k_{2}(x, y)$. Let $l^{\prime}$ be a simple edge path without chords containing $[u v]$ and having length $\geq 5$. Then $x$ or $y$ is a vertex of $l^{\prime}$ (otherwise, $W / N(x y)$ is a smaller example). We may assume $x$ is a vertex of $l^{\prime}$. By Claim 4.3, $y \in l k_{2}(u, v)$. This is impossible as [yv] would be a chord in $l$.

Claim 4.6 If $s \neq y$ and $t \neq x$ are vertices of $l$ adjacent to $x$ and $y$ respectively, then there is not an odd labeled edge at $s$ and an odd labeled edge at $t$.

Proof. Otherwise, say $[s u]$ and $[t v]$ have odd labels. By Claims 4.2 and $4.4 u \neq v$. By Claim 4.3, $u, v \in l k_{2}(x, y)$. Assume $l_{s}$ and $l_{t}$ are circuits without chords and of length $\geq 5$ containing [su] and [tv] respectively. By Claim $4.3, l_{s}$ and $l_{t}$ contain $y$ and $x$ respectively. Hence the paths (suy) and $(t v x)$ are contained in $l_{s}$ and $l_{t}$ 
respectively. Since there is no edge from $t$ to $s$, Claim 4.3 implies that $v \in l k_{2}(s, u)$. By Claim $4.3, l_{t}$ contains $s$ or $u$ and so the vertex of $l_{t}$ following the path $(t v x)$ must be $s$ or $u$, but this is impossible as both are connected to $v$ by an edge (creating a chord in $l_{t}$ ).

We can now complete the reduction.

Case 1. Suppose the only odd labeled edge of $V^{\prime}$ is $[x y]$.

Then say (xyst) is a subpath of $l$. Let $\sigma^{\prime}$ be a simplex of $V^{\prime}$ containing $\{s, t\}$ such that $\left\langle\sigma^{\prime}\right\rangle$ is conjugate to $\langle\sigma\rangle$ for some simplex $\sigma$ of $V$. By Lemma 18, the quotient of $W$ by $N\left(\{s t\} \cup\left(\sigma^{\prime}-\{s, t\}\right)\right)$ is an even Coxeter group. A diagram for this group is obtained from $V^{\prime}$ by identifying $s$ and $t$ and removing the vertices of $\sigma^{\prime}-\{s, t\}$. This diagram contains a circuit of length $\geq 4$, with odd labeled edge $[x y]$ and no chords. This diagram is smaller than $V^{\prime}$, contradicting Proposition 23 or the minimality of $V^{\prime}$.

Case 2. Suppose $V^{\prime}$ contains exactly two odd labeled edges.

Say (uxyst) is a subpath of $l$ and $[u v]$ is an odd labeled edge. By Claim 4.3, $v \in l k_{2}(x, y)$. If $[c d]$ is an edge of $l$ such that $\{c, d\} \cap\{u, x, y\}=\emptyset$, and $\sigma^{\prime}$ is a simplex of $V^{\prime}$ containing $[c d]$ such that $\left\langle\sigma^{\prime}\right\rangle$ is conjugate to $\langle\sigma\rangle$ for some simplex $\sigma$ of $V$, then $\sigma^{\prime}$ must contain $v$ (otherwise, the quotient of $W$ by $N\left(\{c d\} \cup\left(\sigma^{\prime}-\{c, d\}\right)\right.$ ) gives a smaller example). In particular, $v$ is connected to each vertex of $l$ by an edge.

Let $l^{\prime}$ be a circuit in $V^{\prime}$ of length $\geq 5$, containing [uv], and without chords. Then $l \cap l^{\prime}=\{u, y\}$. Note that (uvy) is a subpath of $l^{\prime}$. Let $\sigma^{\prime}$ be a simplex of $V^{\prime}$ containing $[s t v]$ such that $\left\langle\sigma^{\prime}\right\rangle$ is conjugate to $\langle\sigma\rangle$ for some simplex $\sigma$ of $V$. Then $u, x, y \notin \sigma^{\prime}$ and the only vertex of $l^{\prime}$ in $\sigma^{\prime}$ is $v$. A diagram for the even Coxeter group $W / N\left(\sigma^{\prime}-\{v\}\right)$ is obtained from $V^{\prime}$ by removing the vertices of $\sigma^{\prime}-\{v\}$. But this diagram contains a faithful copy of $l^{\prime}$ and so contradicts the minimality of $V^{\prime}$. The proof of Proposition 4 is complete.

\section{REFERENCES}

[1] P. Bahls, Even Rigidity in Coxeter Groups, Ph.D. Thesis, Vanderbilt University, 2002.

[2] R. E. Borcherds Coxeter Groups, Lorentzian Lattices and K3 Surfaces, IMRN 19 (1998), 1011-1031. MR.1654763 (2000a:20088)

[3] B. Brink and R. Howlett, Normalizers of Parabolic Subgroups in Coxeter Groups, Inventiones Mathematicae 136 (1999), 323-351. MR1688445 (2000b:20048)

[4] N. Bourbaki, Groupes et Algebres de Lie Chapters IV-VI, Hermann, Paris, 1968. MR0240238 $(39: 1590)$

[5] N. Brady, J. P. McCammond, B. Mühlherr and W. D. Neumann, Rigidity of Coxeter Groups and Artin Groups, Geom. Dedicata 94 (2002), 91-109. MR1950875 (2004b:20052)

[6] V. V. Deodhar, On Root Systems of a Coxeter Group, Comm. Algebra 10 (1982), 611-630. MR.0647210 (83j:20052a)

[7] M. Mihalik and S. Tschantz, Visual Decompositions of Coxeter Groups, Preprint.

[8] M. Mihalik, J. Ratcliffe and S. Tschantz, Matching Theorems for Systems of a Finitely Generated Coxeter Group, to appear in Algebraic and Geometric Topology.

[9] J. P. Serre, Trees, Springer-Verlag, New York (1980). MR0607504 (82c:20083)

[10] J. Rotman, An Introduction to the Theory of Groups, 4th ed., Graduate Texts in Mathematics 148, Springer Verlag, 1995. MR1307623 (95m:20001)

Department of Mathematics, Vanderbilt University, 1516 Stevenson Center, NashVILLe, Tennessee 37240 